\title{
TECHNIQUES FOR DETERMINING EQUALITY OF THE MAXIMUM NULLITY AND THE ZERO FORCING NUMBER OF A GRAPH*
}

\author{
DEREK YOUNG ${ }^{\dagger}$
}

\begin{abstract}
It is known that the zero forcing number of a graph is an upper bound for the maximum nullity of the graph (see [AIM Minimum Rank - Special Graphs Work Group (F. Barioli, W. Barrett, S. Butler, S. Cioabă, D. Cvetković, S. Fallat, C. Godsil, W. Haemers, L. Hogben, R. Mikkelson, S. Narayan, O. Pryporova, I. Sciriha, W. So, D. Stevanović, H. van der Holst, K. Vander Meulen, and A. Wangsness). Linear Algebra Appl., 428(7):1628-1648, 2008]). In this paper, we search for characteristics of a graph that guarantee the maximum nullity of the graph and the zero forcing number of the graph are the same by studying a variety of graph parameters that give lower bounds on the maximum nullity of a graph. In particular, we introduce a new graph parameter which acts as a lower bound for the maximum nullity of the graph. As a result, we show that the Aztec Diamond graph's maximum nullity and zero forcing number are the same. Other graph parameters that are considered are a Colin de Verdiére type parameter and vertex connectivity. We also use matrices, such as a divisor matrix of a graph and an equitable partition of the adjacency matrix of a graph, to establish a lower bound for the nullity of the graph's adjacency matrix.
\end{abstract}

Key words. Maximum nullity, Zero forcing number, Nullity of a graph, Strong Arnold Property, Equitable partition, Equitable decomposition.

AMS subject classifications. 05C50, 15A18.

1. Introduction. The maximum nullity over the set of matrices described by a graph has been well studied (see $[3,5,13,17])$. There are graph parameters that allow us to bound the maximum nullity. For some graphs, these bounds are enough to determine the maximum nullity. Unfortunately, the bounds available are not enough to determine the maximum nullity for all graphs. The zero forcing number, described in detail below, is an upper bound for maximum nullity. The problem of characterizing graphs for which the maximum nullity of the graph is equal to zero forcing number of the graph was first posed in [3]. While this problem is still open, there are many families of graphs that have their maximum nullity equal to their zero forcing number. A list of families of graphs having this property can be found in [18] including trees, cycles, complete graphs, complete bipartite graphs, completely subdivided graphs, and graphs with less than eight vertices. Even though determining the zero forcing number of a graph is NP-complete (see [1, 2]), the zero forcing number for some graphs can be computed using mathematical software. We were able to compute the zero forcing number for some graphs containing 60 vertices. It is worth noting that some graphs containing as many as 100,000 vertices which have small zero forcing number, such as a path, can be computed.

A graph, denoted by $G$, consists of a set $V(G)$ called a vertex set and an edge set $E(G)$ where the edge set consists of two element subsets of the vertex set. For convenience, when $\{v, u\} \in E(G)$ we may drop the brackets and write $v u$. The order of a graph, denoted by $|G|$, is the number of vertices in the graph. The spectrum of a symmetric matrix $A$, denoted by $\operatorname{spec}(A)$, is the multiset of eigenvalues of $A$. The nullity of a symmetric matrix, denoted by null $(A)$, is the number of times zero occurs in $\operatorname{spec}(A)$. The rank of a symmetric matrix $A$, denoted by $\operatorname{rank}(A)$, is the dimension of the vector space spanned by the rows of $A$.

\footnotetext{
*Received by the editors on January 9, 2020. Accepted for publication on March 29, 2021. Handling Editor: Michael Tsatsomeros.

${ }^{\dagger}$ Mount Holyoke College, South Hadley, MA, USA (dyoung@mtholyoke.edu).
} 
The adjacency matrix of a graph $G$, denoted by $A(G)$, is a $(0,1)$-matrix such that the $i j$ entries of the matrix is 1 if and only if $G$ contains the edge $\{i, j\}$. The set of symmetric matrices of a graph $G$ over a field $\mathcal{F}$, denoted by $\mathcal{S}(\mathcal{F}, G)$, is the set of symmetric matrices $A=\left[a_{i j}\right]$ having the same off-diagonal nonzero pattern as $A(G)$ with free diagonal entries $\left(a_{i i} \in \mathcal{F}\right)$. The maximum nullity of a graph $G$ over a field $\mathcal{F}$, denoted by $\mathrm{M}(\mathcal{F}, G)$, is the maximum nullity over $\mathcal{S}(\mathcal{F}, G)$. Let $A \in \mathcal{S}(\mathbb{R}, G)$. Because the diagonal of a matrix $A \in \mathcal{S}(\mathbb{R}, G)$ is unrestricted, all eigenvalues of $A$ are real, the algebraic and geometric multiplicity of $A$ are equal, and the nullity of $A-\lambda I$ is the multiplicity of $\lambda$ as an eigenvalue of $A$. Thus, the maximum multiplicity over matrices in $\mathcal{S}(\mathbb{R}, G)$ is the same as the maximum nullity over $\mathcal{S}(\mathbb{R}, G)$. The minimum rank of a graph $G$ over a field $\mathcal{F}$, denoted by $\operatorname{mr}(\mathcal{F}, G)$, is the minimum rank over $\mathcal{S}(\mathcal{F}, G)$. Whenever the field is not specified, the field is understood to be the real numbers $\mathbb{R}$. Observe that $\operatorname{mr}(\mathcal{F}, G)+\mathrm{M}(\mathcal{F}, G)=|G|$, where $|G|$ is defined to be the number of vertices in the graph $G$. As a result, value of $\operatorname{M}(\mathcal{F}, G)$ gives the solution to the minimum rank problem of the graph $G$. See [13] for a discussion on the motivation of the minimum rank problem.

Let $Z$ be a subset of $V(G)$ such that every vertex in $Z$ is colored blue and all other vertices are colored white. The color change rule for zero forcing is: A blue vertex can change a white vertex blue if the white vertex is the only white vertex adjacent to the blue vertex. (Vertices $v$ and $u$ are said to be adjacent if and only if $\{v, u\} \in E(G)$.) In this case, we say that the blue vertex forced the white vertex blue. A zero forcing set is a subset of $V(G)$ such that after applying the color change rule until no more changes are possible, all vertices in $G$ are colored blue. The zero forcing number of a graph $G$, denoted by $\mathrm{Z}(G)$, is the minimum cardinality over all zero forcing sets. A chronological list of forces is a sequence of forces performed in the given order. The term zero forcing refers to forcing entries in the null vector to be zero, which leads to the relationship that the maximum nullity of a graph is bounded above by the zero forcing number of the graph.

Proposition 1.1 ([3, Proposition 2.4]). Let $G$ be a graph and let $\mathcal{F}$ be a field. Then

$$
\mathrm{M}(\mathcal{F}, G) \leq \mathrm{Z}(G) .
$$

Note that $A(G)-\lambda I$ with $\lambda \in \mathbb{Z}$ can be viewed as a matrix over any field $\mathcal{F}$. Thus, $A(G)-\lambda I \in \mathcal{S}(\mathcal{F}, G)$. When we view $A \in \mathcal{F}^{n \times n}$ we write $\operatorname{rank}(\mathcal{F}, A)$. An optimal matrix over a field $\mathcal{F}$ is a matrix $A \in \mathcal{S}(G)$ such that $\operatorname{rank}(\mathcal{F}, A)=\operatorname{mr}(\mathcal{F}, G)$. We say that an integer matrix $A \in \mathcal{S}(\mathcal{F}, G)$ that has entries $-1,0,1$ on the off-diagonal is universally optimal if for all fields $\mathcal{F}, \operatorname{rank}(\mathcal{F}, A)=\operatorname{mr}(\mathcal{F}, G)$. The minimum rank of a graph $G$ is said to be field independent if for all fields $\mathcal{F}, \operatorname{mr}(\mathcal{F}, G)=\operatorname{mr}(G)$. The minimum rank problem over fields other than the real numbers was studied as early as 2004 by Wayne Barrett, Hein van der Holst, and Raphael Loewy in [8]. In 2009, DeAlba et al. [12] used universally optimal matrices to establish minimum rank field independence for many graphs listed in [18].

Proposition 1.2 ([12, Corollary 2.3]). If $A \in \mathbb{Z}^{n \times n}$, then $\operatorname{rank}\left(\mathbb{Z}_{p}, A\right) \leq \operatorname{rank}(A)$ for every prime $p$.

Corollary 1.3. Let $G$ be a graph having the property that for some $\lambda \in \mathbb{Z}, \operatorname{rank}(A(G)-\lambda I)=|G|-$ $\mathrm{Z}(G)$, or equivalently, null $(A(G)-\lambda I)=\mathrm{Z}(G)$. Then the minimum rank of $G$ is field independent and $A(G)-\lambda I$ is universally optimal, and $\mathrm{M}(\mathcal{F}, G)=\mathrm{Z}(G)$ for all fields $\mathcal{F}$.

Proof. By Proposition 1.1, $|G|-\operatorname{mr}(\mathcal{F}, G)=\mathrm{M}(\mathcal{F}, G) \leq \mathrm{Z}(G)$ and by Proposition 1.2, we have

$$
\operatorname{rank}(\mathcal{F}, A(G)-\lambda I) \leq \operatorname{rank}(A(G)-\lambda I),
$$

so $\operatorname{null}(A(G)-\lambda I) \leq \operatorname{null}(\mathcal{F}, A(G)-\lambda I)$. It follows that

$$
\mathrm{Z}(G) \geq \mathrm{M}(\mathcal{F}, G) \geq \operatorname{null}(\mathcal{F}, A(G)-\lambda I) \geq \operatorname{null}(A(G)-\lambda I)=\mathrm{Z}(G) .
$$


297 Techniques for determining equality of the maximum nullity and the zero forcing number of a graph

Therefore, $\operatorname{mr}(\mathcal{F}, G)=\operatorname{rank}(\mathcal{F}, A(G)+\lambda I)=|G|-\mathrm{Z}(G)$ which shows that $G$ has field independent minimum rank and $A(G)+\lambda I$ is universally optimal.

OBSERVATION 1.4. Let $G$ be a graph. If there exists a prime $p$ such that $\operatorname{mr}\left(\mathbb{Z}_{p}, G\right) \neq \operatorname{mr}(G)$, then $G$ does not have field independent minimum rank.

A generalized Petersen Graph, denoted by $P(n, k)$, is a graph having a labeled vertex set

$$
\left\{u_{0}, u_{1}, \ldots u_{n-1}, v_{0}, v_{1}, \ldots, v_{n-1}\right\}
$$

and edge set $\left\{\left\{u_{i} u_{i+1} \bmod n\right\},\left\{v_{i} v_{i+k \bmod n}\right\},\left\{u_{i} v_{i}\right\}: i=0,1,2, \ldots, n-1\right\}$, for $n \geq 3$ and $k$ a positive integer less than $\left\lfloor\frac{n}{2}\right\rfloor$ (see [22]). In [4], the adjacency matrix was used to show that the maximum nullity is equal to the zero forcing number for certain generalized Petersen graphs.

TheOREM 1.5 ([4, Theorem 2.4]). Let $r$ be a positive integer. Then

$$
\mathrm{M}(P(15 r, 2))=\mathrm{Z}(P(15 r, 2))=6 \quad \text { and } \quad \mathrm{M}(P(24 r, 5))=\mathrm{Z}(P(24 r, 5))=12,
$$

and the maximum nullity is attained by the adjacency matrix.

Corollary 1.6. Let $r$ be a positive integer. Then $P(15 r, 2)$ and $P(24 r, 5)$ have field independent minimum rank with universally optimal matrices. Moreover, for all fields $\mathcal{F}$,

$$
\mathrm{M}(\mathcal{F}, P(15 r, 2))=\mathrm{Z}(P(15 r, 2)) \quad \text { and } \quad \mathrm{M}(\mathcal{F}, P(24 r, 5))=\mathrm{Z}(P(24 r, 5)) .
$$

The Cartesian product of the graphs $G$ and $H$, denoted by $G \square H$, has vertex set $\{(v, w) \mid v \in V(G), w \in$ $V(H)\}$ and edge set

$$
\left\{\left(v_{1}, w_{1}\right)\left(v_{2}, w_{2}\right) \mid\left(v_{1}=v_{2} \text { and } w_{1} w_{2} \in E(H)\right) \text { or }\left(v_{1} v_{2} \in E(G) \text { and } w_{1}=w_{2}\right)\right\}
$$

Theorem 1.7 ([3, Theorem 3.8]). Let $k \geq 3$. Then $\mathrm{M}\left(C_{k} \square P_{t}\right)=\mathrm{Z}\left(C_{k} \square P_{t}\right)=\min \{k, 2 t\}$.

ExAMPLE 1.8. By Theorem 1.7, $\mathrm{M}\left(C_{7} \square P_{2}\right)=4$ which implies $\operatorname{mr}\left(C_{7} \square P_{2}\right)=10$. By computation via SageMath (see [23]), there does not exist a matrix in $\mathcal{S}\left(\mathbb{Z}_{2}, C_{7} \square P_{2}\right)$ having rank equal to 10 . Therefore by Observation 1.4, $C_{7} \square P_{2}$ does not have field independent minimum rank.

Example 1.8 shows that the generalized Petersen graphs do not have field independent minimum rank since $C_{7} \square P_{2}$ is isomorphic to $P(7,1)$. It is known that $C_{n} \square P_{t}$ does not have field independent minimum rank (see [12, Example 3.5]).

2. An application of the nullity of a graph. In this section, we introduce a new combinatorial approach for determining the nullity of the adjacency matrix of a graph, which naturally acts as a lower bound for the maximum nullity of the graph. We use the nullity of a graph to give a combinatorial proof of the nullity of the adjacency matrix for the Aztec diamond graphs. This result is used to show that the maximum nullity and zero forcing number of Aztec diamond graphs are the same. We also give results for some circulant graphs.

A general graph is a graph that may contain loops (edges of the form $v v$ ) and/or multi-edges (two edges containing the same vertices $u$ and $v$ are called multi-edges). Let $G$ be a general graph and let $v, u \in V(G)$. The neighborhood of $v$ in a general graph $G$, denoted by $N_{G}(v)$, is a multiset containing vertices of $V(G)$ such that $k$ copies of $u$ are in $N_{G}(v)$ if and only if there are $k$ copies of $u v$ in $E(G)$. Let $X$ and $Y$ be multisets 

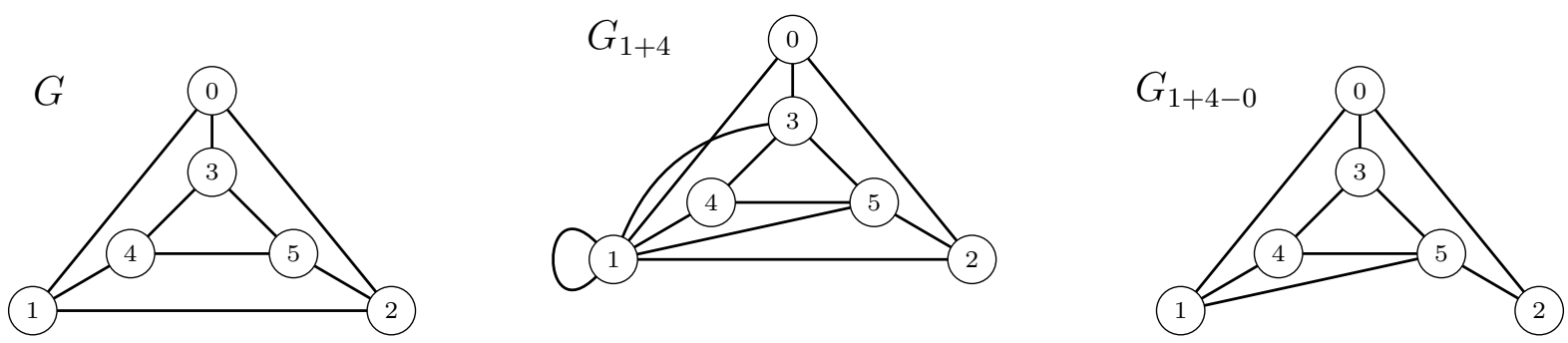

Figure 1: This shows the graph $G_{1+4-0}$.

containing elements of $V(G)$. The general graph $G_{v+X}$ is obtained from $G$ by adding one edge $v w$ for each $w \in N_{G}(x)$ and for every $x \in X$ (see Figure 1). Let $v \in X$ and $y \in Y$. Suppose $N_{G_{v+X}}(y) \subseteq N_{G_{v+X}}(v)$. Then the general graph $G_{v+X-Y}$ is obtained from $G_{v+X}$ by deleting one edge $v w$ for each $w \in N_{G_{v+X}}(y)$ and for every $y \in Y$ (see Figure 1). In the case that $X$ and $Y$ consist of a single vertex $x$ or $y$, we write $G_{v+x}$ or $G_{v+x-y}$.

We define a color change rule as follows: In a graph $G$, having each vertex colored red or white, a white vertex $u$ can be colored red if there exists a white vertex $v$ and multisets of white vertices $X, Y$ such that

1. $u \notin\{v\} \cup X \cup Y$, and

2. $N_{G_{u+U_{k}}}(u)=N_{G_{v+X-Y}}(v)$

for some nonnegative integer $k$ and the multiset $U_{k}$ containing $k$ copies of $u$ (whenever $k=0, U_{k}$ is the empty set and $\left.N_{G_{u+U_{k}}}(u)=N_{G}(u)\right)$. In this case, we say that $u$ can be colored red by $(v, X, Y, k)$.

ExAmple 2.1. Figure 1 illustrates the process of creating $G_{1+4-0}$. Moreover, vertices 1 and 3 have the same neighborhood in $G_{1+4-0}$, so vertex 3 can be colored red in $G$ by $(1,\{4\},\{0\}, 0)$. We can also color vertex 5 red. Consider the general graph $G_{1+4-2}$ in which vertices 1 and 5 have the same neighborhood in $G_{1+4-2}$.

A set $\left\{v_{1}, v_{2}, \ldots, v_{t}\right\}$ of red vertices is called a red set, denoted by $\mathcal{R}$, if the vertices $v_{1}, v_{2}, \ldots, v_{t}$ can be sequentially colored red. The nullity of a graph $G$, denoted by $\mathfrak{N}(G)$, is the maximum cardinality over the set of all red sets.

OBservation 2.2. Let $u, v$ be white vertices of $V(G), X$ and $Y$ be multisets containing white vertices of $V(G)$, and $k$ be a nonnegative integer. Then $u$ can be colored red by $(v, X, Y, k)$ if and only if

$$
(k+1) \cdot \operatorname{row}_{A(G)}(u)=\operatorname{row}_{A(G)}(v)+\sum_{x \in X} \operatorname{row}_{A(G)}(x)-\sum_{y \in Y} \operatorname{row}_{A(G)}(y) .
$$

TheOREM 2.3. Let $G$ be a simple graph. Then $\mathfrak{N}(G)=\operatorname{null}(A(G))$.

Proof. Let $G$ be a graph with all vertices initially colored white. Suppose that at some stage the vertices $u_{1}, u_{2}, \ldots, u_{q-1}$ have been sequentially colored red, the remaining vertices colored white, and that

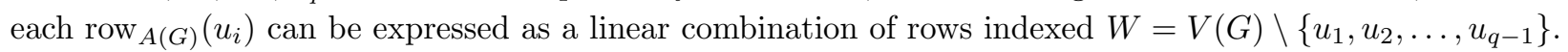
Suppose that $v$ and the vertices of $X, Y$ are white and $u_{q}$ can be colored red by $(v, X, Y, k)$. We show that $\operatorname{row}_{A(G)}\left(u_{i}\right)$ for $i=1,2, \ldots, q$ can each be expressed as a linear combination of rows indexed by $W^{\prime}=W \backslash\left\{u_{q}\right\}$. 
299 Techniques for determining equality of the maximum nullity and the zero forcing number of a graph

Let $W^{\prime}=\left\{w_{1}, w_{2}, \ldots, w_{\ell}\right\}$. By $(1)$, row $_{A(G)}\left(u_{q}\right)$ can be expressed as a linear combination of rows indexed by $W^{\prime}$. We know that $\operatorname{row}_{A(G)}\left(u_{i}\right)$ can be expressed as a linear combination of the rows associated with the vertices in $W=W^{\prime} \cup\left\{u_{q}\right\}$. By substituting the expression for $\operatorname{row}_{A(G)}\left(u_{q}\right)$ into that for row $A(G)\left(u_{i}\right)$, we see that row $\operatorname{rag}_{(G)}\left(u_{i}\right)$ is a linear combination of rows associated with vertices in $W^{\prime}$. At the conclusion of this process $\operatorname{rank}(A(G)) \leq n-\mathfrak{N}(G)$, so $\mathfrak{N}(G) \leq \operatorname{null}(A(G))$.

Let $W$ be a set of linearly independent rows of $A(G)$ that forms a basis for the row space of $A(G)$. Let $r=|W|$ and let $v_{1}, v_{2}, \ldots, v_{r}$ be the vertices associated with these rows. Then each row not in $W$, $\operatorname{row}_{A(G)}\left(v_{j}\right)$ with $j>r$, can be written as

$$
\frac{c_{1}}{d_{1}} \operatorname{row}_{A(G)}\left(v_{1}\right)+\frac{c_{2}}{d_{2}} \operatorname{row}_{A(G)}\left(v_{2}\right)+\cdots+\frac{c_{r}}{d_{r}} \operatorname{row}_{A(G)}\left(v_{r}\right),
$$

where $c_{i}, d_{i} \in \mathbb{Z}$ and $d_{i}>0$ for $i=1, \ldots, r$. By letting $d=\operatorname{lcm}\left(d_{1}, d_{2}, \ldots, d_{r}\right)$, we can write

$$
d \cdot \operatorname{row}_{A(G)}\left(v_{j}\right)=c_{1} s_{1} \operatorname{row}_{A(G)}\left(v_{1}\right)+c_{2} s_{2} \operatorname{row}_{A(G)}\left(v_{2}\right)+\cdots+c_{r} s_{r} \operatorname{row}_{A(G)}\left(v_{r}\right)
$$

where $s_{i}=d / d_{i} \in \mathbb{Z}$. Fix $v_{j}$ corresponding to a row in $W$. Let $\ell \in\{1,2, \ldots, r\}$ such that $c_{\ell} s_{\ell}>0$. Let $X$ be the multiset of vertices consisting of $c_{\ell} s_{\ell}-1$ copies of $v_{\ell}$ and $c_{i} s_{i}$ copies of $v_{i}$ for $i \neq \ell$ and $c_{i} s_{i}>0$ and let $Y$ be the multiset of vertex consisting of $c_{i} s_{i}$ copies of $v_{i}$ for $c_{i} s_{i}<0$. Then $v_{j}$ can be colored red by $\left(v_{\ell}, X, Y, d-1\right)$. This implies $\mathfrak{N}(G) \geq n-r \geq n-\operatorname{rank}(A(G))=\operatorname{null}(A(G))$.

Corollary 2.4. Let $G$ be a bipartite graph with independent sets $\mathbf{B}$ and $\overline{\mathbf{B}}$ such that $|\mathbf{B}|=|\overline{\mathbf{B}}|$. Let $\mathcal{R} \subseteq \mathbf{B}$ be a red set such that every vertex in $\mathcal{R}$ is colored with some $(v, X, Y, k)$ where $\{v\} \cup X \cup Y$ contains only vertices from $\mathbf{B}$. Then $2|\mathcal{R}| \leq \operatorname{null}(A(G))$.

The Aztec diamond of order $r$ is a diamond shape configuration of $2 \mathrm{r}(\mathrm{r}+1)$ unit squares, as illustrated in Figure 2. The Aztec diamond graph of order $r$, denoted by $\mathrm{AD}_{r}$, is the graph such that vertices $v, u \in V\left(\mathrm{AD}_{r}\right)$ are adjacent if and only if squares $v$ and $u$ share an edge in the Aztec diamond of order $r$. The vertices of $\mathrm{AD}_{r}$ are labeled by ordered pairs $(i, j)$ where $1 \leq i, j \leq 2 r, r+1 \leq i+j \leq 3 r+1$, and $0 \leq|j-i| \leq r$.
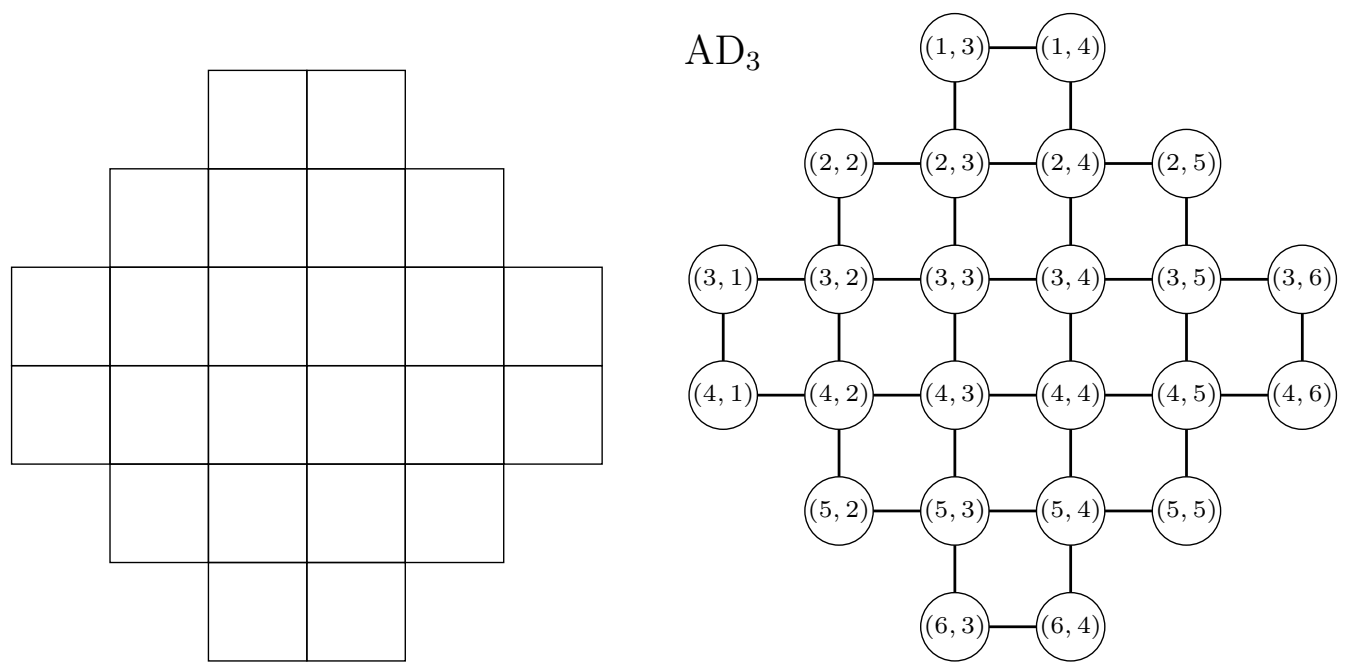

Figure 2: The Aztec diamond of order 3 and the Aztec diamond graph $\mathrm{AD}_{3}$. 
Proposition 2.5. Let $G$ be an Aztec diamond graph $\mathrm{AD}_{r}$. Then $\mathrm{Z}(G) \leq 2 r$.

Proof. We show that the set $Z=\{(1, r),(2, r-1),(3, r-2), \ldots,(r, 1)\} \cup\{(1, r+1),(2, r+2),(3, r+$ $3), \ldots,(r, 2 r)\}$ is a zero forcing set. For $i \in\{1,2, \ldots, r\}$ in order $(i, j)$ can force $(i+1, j)$ as long as $(i, j)$ and $(i+1, j)$ exist.

ThEOREM 2.6. Let $\mathrm{AD}_{r}$ be an Aztec diamond graph of order $r$ and $\mathcal{F}$ be an arbitrary field. Then

$$
\mathrm{M}\left(\mathcal{F}, \mathrm{AD}_{r}\right)=\mathrm{Z}\left(\mathrm{AD}_{r}\right)=2 r
$$

and field independent minimum rank is established with the universally optimal matrix $A(G)$.

Proof. Let $D_{\ell}=\{(i+\ell, r+2+\ell-i) \mid 1 \leq i \leq r+1\}$ for $0 \leq \ell \leq r-1$. Note that the $D_{\ell}$ are independent sets and disjoint. Let $\mathbf{B}=D_{0} \cup D_{1} \cup D_{2} \cup \cdots \cup D_{(r-1)}$. We show that $r$ vertices of $\mathbf{B}$ can be colored red by other vertices of $\mathbf{B}$. The vertex $(r+1,1)$ in the set $D_{0}$ can be colored red by $\left((r, 2),\left\{(i, j) \in D_{0} \mid i<r, j\right.\right.$ is even $\},\left\{(i, j) \in D_{0} \mid i<r, j\right.$ is odd $\left.\}, 0\right)$. See Figure 3 for an example. Using a similar argument, each $D_{\ell}$ has a vertex that can be colored red using only vertices from $D_{\ell}$. Since $\mathbf{B}$ is partitioned into $r$ sets $D_{\ell}$, a total of $r$ vertices that can be colored red. By Corollary $2.4,2 r \leq$ null( $\left.\mathrm{AD}_{r}\right)$. By Theorem 2.3 and Proposition 2.5, $2 r \leq \operatorname{null}\left(A\left(\mathrm{AD}_{r}\right)\right) \leq \mathrm{M}\left(\mathrm{AD}_{r}\right) \leq \mathrm{Z}\left(\mathrm{AD}_{r}\right) \leq 2 r$.

A circulant graph, denoted by $\operatorname{Circ}[n, S]$, is a graph with vertex set $\{0,1, \ldots, n-1\} \subseteq \mathbb{Z}$ and a connection set $S \subseteq\left\{1,2, \ldots, \frac{n}{2}\right\} \subseteq \mathbb{Z}$, where the edge set of $\operatorname{Circ}[n, S]$ is precisely $\left.\{\{i, i \pm s\}: s \in S\}\right\}$ with arithmetic performed modulo $n$ (see Figure 4). For any $a \in[n]$, the graphs $\operatorname{Circ}[n, S]$ and $\operatorname{Circ}[n, a S]$ are isomorphic whenever $a$ and $n$ are relatively prime. Thus, if there exists $b \in S$ such that $\operatorname{gcd}(b, n)=1$, then $1 \in b^{-1} S$ and $\operatorname{Circ}[n, S] \cong \operatorname{Circ}\left[n, b^{-1} S\right]$. For simplicity, all circulant graphs considered here have 1 in the connection set.

$\mathrm{AD}_{3(3,2)+(1,4)}$

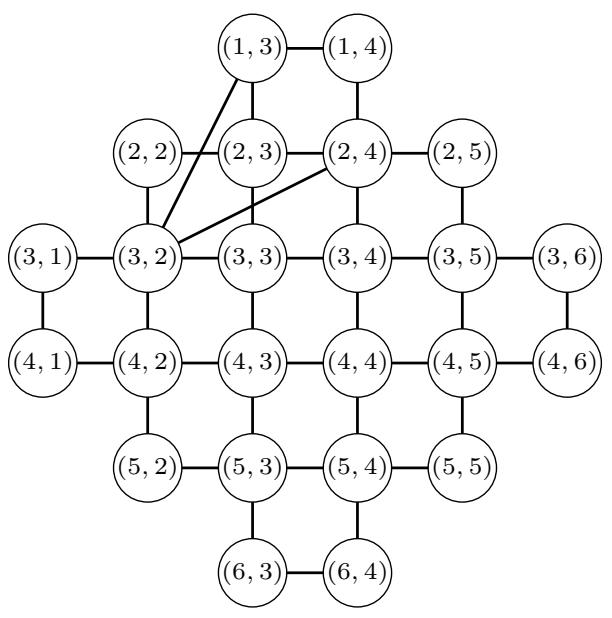

$\mathrm{AD}_{3(3,2)+(1,4)-(2,3)}$

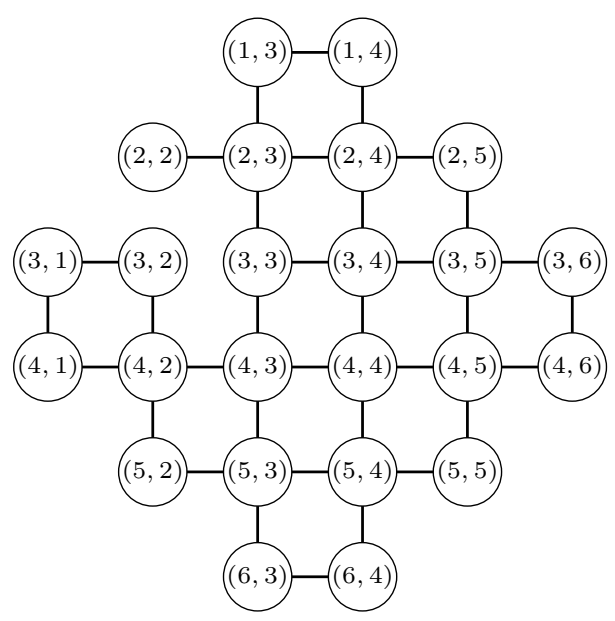

Figure 3: Coloring $(4,1)$ red with $((3,2),\{(1,4)\},\{(2,3)\}, 0)$ in the Aztec diamond graph $\mathrm{AD}_{3}$. 
301 Techniques for determining equality of the maximum nullity and the zero forcing number of a graph
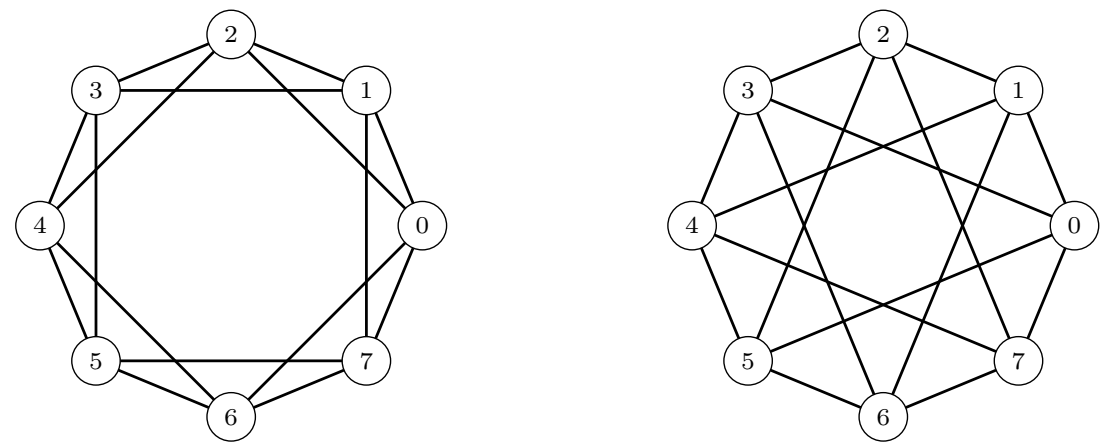

Figure 4: The circulants $\operatorname{Circ}[8,\{1,2\}]$ and $\operatorname{Circ}[8,\{1,3\}]$.

Proposition 2.7. Let $n$ be a multiple of 8 . Then,

$$
\mathrm{Z}\left(\operatorname{Circ}\left[n,\left\{1, \frac{n}{2}-1\right\}\right]\right) \leq \frac{n}{2}+2 .
$$

Proof. Let $G=\operatorname{Circ}\left[n,\left\{1, \frac{n}{2}-1\right\}\right]$. Then $Z=\left\{0,1,2, \ldots, \frac{n}{2}, n-1\right\}$ is a zero forcing set with forces $0 \rightarrow n / 2+1,1 \rightarrow n / 2+2, \cdots, n / 2-3 \rightarrow n-2$. This shows that $\mathrm{Z}(G) \leq \frac{n}{2}+2$

THEOREM 2.8. Let $n$ be a multiple of 8 . Then,

$$
\mathrm{M}\left(\operatorname{Circ}\left[n,\left\{1, \frac{n}{2}-1\right\}\right]\right)=\mathrm{Z}\left(\operatorname{Circ}\left[n,\left\{1, \frac{n}{2}-1\right\}\right]\right)=\frac{n}{2}+2,
$$

and field independent minimum rank is established with the universally optimal matrix $A(G)$.

Proof. First note that $G$ is bipartite with partite set $\mathbf{B}=\left\{2 k \mid 0 \leq k \leq \frac{n}{2}-1\right\}$ and $\overline{\mathbf{B}}=\{2 k+1 \mid 0 \leq$ $\left.k \leq \frac{n}{2}-1\right\}$. We show that $\frac{n}{4}+1$ vertices from $\mathbf{B}$ can be colored red using only white vertices of B. Note that for every vertex $v$ in $\left\{0,1,2, \ldots, \frac{n}{2}-1\right\}, v$ is adjacent to $v+1, v-1, v+\frac{n}{2}-1, v+\frac{n}{2}+1$, and $v+\frac{n}{2}$ is adjacent to $v+\frac{n}{2}+1, v+\frac{n}{2}-1, v+\frac{n}{2}+\frac{n}{2}-1 \equiv v-1 \bmod n, v+\frac{n}{2}+\frac{n}{2}+1 \equiv v+1 \bmod n$. Hence, $N_{G}(v)=N_{G}\left(v+\frac{n}{2}\right)$ and $v$ can be colored red by $\left(v+\frac{n}{2}, \emptyset, \emptyset, 0\right)$ where $v \in\left\{0,2,4, \ldots, \frac{n}{2}-2\right\}$. This shows that $\frac{n}{4}$ vertices from $\mathbf{B}$ can be colored red. The vertex $\frac{n}{2}$ can be colored red by $\left(\frac{n}{2}+2,\left\{2 i: 2 \mid i\right.\right.$ and $\frac{n}{2}+2<$ $2 i \leq n-1\},\left\{2 i: 2 \nmid i\right.$ and $\left.\left.\frac{n}{2}+2<2 i \leq n-1\right\}, 0\right)$. Hence, the vertices of $\left\{0,2,4, \ldots, \frac{n}{2}\right\}$ can be colored red with the vertices $\mathbf{B} \backslash\left\{0,2,4, \ldots, \frac{n}{2}\right\}$. By Corollary $2.4,2\left(\frac{n}{4}+1\right)=\frac{n}{2}+2 \leq \mathfrak{N}(G)$. So by Theorem 2.3 and by Proposition 2.7

$$
\frac{n}{2}+2 \leq \operatorname{null}(A(G)) \leq \mathrm{M}(G) \leq \mathrm{Z}(G) \leq \frac{n}{2}+2
$$

3. An application of the Strong Arnold Property (SAP). In this section, we use the Colin de Verdière type parameter $\xi$ to show that the maximum nullity and zero forcing number of various families of graphs are equal.

A matrix $A \in \mathcal{S}(G)$ has the $\mathrm{SAP}$ if there does not exist a nonzero symmetric matrix $X$ having the following three properties: (1) $A X=0,(2) A \circ X=0,(3) I \circ X=0$ where $\circ$ is the Hadamard (entrywise) product. The Colin de Verdière type parameter associated with the maximum nullity is

$$
\xi(G)=\max \{\operatorname{null}(A) \mid A \in \mathcal{S}(G) \text { and } A \text { has the SAP }\} .
$$

Clearly, $\xi(G) \leq \mathrm{M}(G) \leq \mathrm{Z}(G)$ for all graphs $G$. The parameter $\xi$ was introduced in 2005 in [6] to gain more insight on the minimum rank of a graph. 
Example 3.1. Using SageMath (see [16]), $A\left(C_{8} \square P_{3}\right)$ has the SAP and null $\left(A\left(C_{8} \square P_{3}\right)\right)=6$. By Theorem 1.7, $\mathrm{M}\left(C_{8} \square P_{3}\right)=\mathrm{Z}\left(C_{8} \square P_{3}\right)=6$. Therefore, $\xi\left(C_{8} \square P_{3}\right)=\mathrm{M}\left(C_{8} \square P_{3}\right)=\mathrm{Z}\left(C_{8} \square P_{3}\right)=6$.

An edge contraction of a graph $G$ is defined to be a deletion of two adjacent vertices $v_{1}$ and $v_{2}$ and an insertion of a vertex $u$ such that $u v \in E(G)$ if and only if $v v_{1} \in E(G)$ or $v v_{2} \in E(G)$. A graph $H$ is a minor of a graph $G$ if $H$ can be constructed from $G$ by performing edge deletions, vertex deletions, and/or contractions. We write $H \preceq G$ when $H$ is a minor of $G$. Note that $G \preceq G^{\prime} \preceq G^{\prime \prime}$ implies $G \preceq G^{\prime \prime}$.

OBservation 3.2. Let $3 \leq k \leq n$ and $1 \leq r \leq t$. Then $C_{k} \square P_{r} \preceq C_{n} \square P_{t}$.

Theorem 3.3 ([6, Corollary 2.5]). If $H$ is a minor of $G$, then $\xi(H) \leq \xi(G)$.

Definition 3.4. Let $H$ be a minor of $G$. We say that $H$ is a zero forcing minor of $G$ if $\mathrm{Z}(G) \leq \mathrm{Z}(H)$.

TheOREM 3.5. Let $H$ be a zero forcing minor of $G$ such that $\xi(H)=\mathrm{Z}(H)$. Then $\xi(G)=\mathrm{M}(G)=$ $\mathrm{Z}(G)=\mathrm{Z}(H)$.

Proof. Given that $H$ is a zero forcing minor, $\mathrm{Z}(G) \leq \mathrm{Z}(H)$. By Theorem 3.3, $\xi(H) \leq \xi(G)$ and it follows that

$$
\mathrm{Z}(H)=\xi(H) \leq \xi(G) \leq \mathrm{M}(G) \leq \mathrm{Z}(G) \leq \mathrm{Z}(H) .
$$

Thus, the parameters $\xi(G), \mathrm{M}(G), \mathrm{Z}(G)$, and $\mathrm{Z}(H)$ are equal.

Corollary 3.6. Let $G=C_{n} \square P_{3}$ such that $8 \leq n$. Then

$$
\xi(G)=\mathrm{M}(\mathcal{F}, G)=\mathrm{Z}(G)=6 .
$$

A $k$-subdivision of an edge, say $u v$, is an operation on a graph in which edge $u v$ is deleted, vertices $v_{1}, v_{2}, \ldots, v_{k}$ and edges $u v_{1}, v_{1} v_{2}, v_{2} v_{3}, \ldots, v_{k} v$ are added. We say the edge $u v$ has been $k$-subdivided. Whenever $k=1$ we simply say that the edge $u v$ has been subdivided. A $k$-subdivision edge insertion on the edges $u v$ and $w x$ is an operation on a graph in which edges $u v$ and $w x$ are $k$-subdivided adding vertices $v_{1}, v_{2}, \ldots, v_{k}$ and $x_{1}, x_{2}, \ldots, x_{k}$, respectively, and edges $v_{1} x_{1}, v_{2} x_{2}, \ldots, v_{k} x_{k}$ are added. The cube graph $Q_{3}$ can be described by an 8-cycle containing a labeled vertex set $\{0,1, \ldots, 7\}$ and added edges $\{\{0,5\},\{1,4\},\{2,7\},\{3,6\}\}$ as shown in Figure 5 .

Proposition 3.7 ([21, Lemma 8]). For the cube graph, $\xi\left(Q_{3}\right)=4=\mathrm{M}(G)=\mathrm{Z}(G)$.

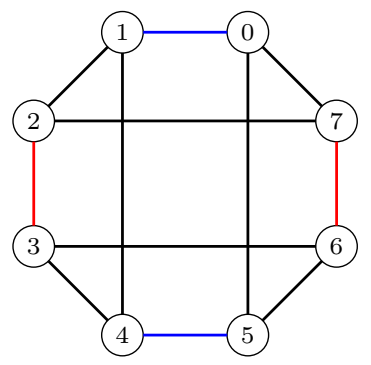

The cube graph

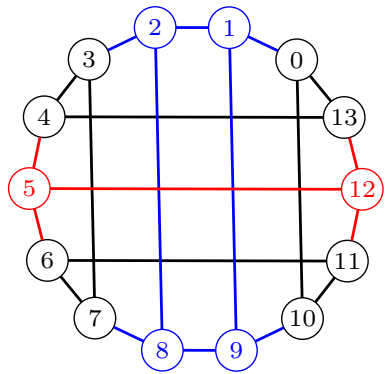

$\operatorname{ECG}(1,2)$

Figure 5: Applying two vertical and one horizontal subdivision edge insertion on the cube graph gives $\operatorname{ECG}(1,2)$. 
303 Techniques for determining equality of the maximum nullity and the zero forcing number of a graph

DEFINITION 3.8 (Extended cube graph). A vertical $k$-subdivision edge insertion on the cube graph is a $k$-subdivision edge insertion on the edges $\{0,1\}$ and $\{4,5\}$. A horizontal $k$-subdivision edge insertion on the cube graph is a $k$-subdivision edge insertion on the edges $\{2,3\}$ and $\{6,7\}$, with the numbering as in Figure 5. An extended cube graph, denoted by $\operatorname{ECG}(t, k)$, is the cube graph with a horizontal $t-$ subdivision edge insertion, a vertical $k$-subdivision edge insertion, and a relabeling around the cycle containing vertex set $\{0,1, \ldots, 7+2(t+k)\}$.

Figure 5 shows $\operatorname{ECG}(1,2)$. Notice that $\operatorname{ECG}(t, k)$ isomorphic to the graph $\operatorname{ECG}(k, t)$. For simplicity, we consider the extended cube graphs with $t \leq k$. The graph ECG $(1,1)$ is called the Bidiakis cube (see [4]). It was shown in [4, Proposition 5.1] that the maximum nullity and zero forcing number of the Bidiakis cube are the same, motivating the creation of the extended cube graphs. Observe that in ECG $(t, k)$, as we draw it, the top endpoints of the vertical edges are $0, \ldots, k+1$, the left endpoints of the horizontal edges are $k+2, \ldots, t+k+3$, the lower endpoints of the vertical edges are $t+k+4, \ldots, t+2 k+5=n-t-3$, and the right endpoints of the horizontal edges are $t+2 k+6, \ldots, 2 t+2 k+7=n-1$.

OBSERVATION 3.9. Let $G$ be a graph constructed from the graph $H$ by performing a subdivision edge insertion. Then $H \preceq G$.

Proposition 3.10. Let $G$ be an extended cube graph $\operatorname{ECG}(t, k)$. Then $\mathrm{Z}(G) \leq 4$.

Proof. Let $n$ be the number of vertices of $G$ and let $r=n-t-3$. The set $\{0, r, r+1, n-1\}$ is a zero forcing set with simultaneous forces

$$
\begin{aligned}
& 0 \rightarrow 1 \rightarrow 2 \rightarrow \cdots \rightarrow k+2, \\
& r \rightarrow r-1 \rightarrow r-2 \rightarrow \cdots \rightarrow r-(k+2)=k+t+3
\end{aligned}
$$

These forcing sequences run simultaneously in parallel, i.e., $0 \rightarrow 1$ and $r \rightarrow r-1$ are simultaneous, etc. After the above forces are completed, the following forces run in parallel

$$
\begin{aligned}
& (k+2) \rightarrow(k+2)+1 \quad \rightarrow \quad(k+2)+3 \rightarrow \cdots \quad \rightarrow \quad(k+2)+t, \\
& (n-1) \rightarrow(n-1)-1 \rightarrow(n-1)-2 \rightarrow \cdots \rightarrow(n-1)-t=2 k+t+6 .
\end{aligned}
$$

Corollary 3.11. Let $G$ be the extended cube graph $\operatorname{ECG}(t, k)$. Then

$$
\xi(G)=\mathrm{M}(G)=\mathrm{Z}(G)=4 .
$$

Proof. Let $H$ be the cube graph. By Proposition 3.7, $\xi(H)=\mathrm{Z}(\mathrm{H})=4$. By Theorem 3.5, $H$ is a zero forcing minor of $G$. Thus, $\xi(G)=\mathrm{M}(G)=\mathrm{Z}(G)=4$ by Theorem 3.5.

Observation 3.12. For positive integer $k$,

$$
\operatorname{Circ}[4 k,\{1,3, \ldots, 2 k-1\}]=K_{2 k, 2 k} \quad \text { and } \quad \operatorname{Circ}[4 k+2,\{1,3, \ldots, 2 k+1\}]=K_{2 k+1,2 k+1} .
$$

Proposition 3.13 and Theorem 4.4 below were found by several groups in 2009 and 2010 but not published. Some of these results were also published in [11]. We state these results and give formal proofs of the results for clarity.

Proposition 3.13 ([15, Proposition 2.1]). Let $G$ be a circulant graph $\operatorname{Circ}[n, S]$ and let $m=\max \{i \mid i \in$ $S\}$. Then $\mathrm{Z}(G) \leq 2 m$. 

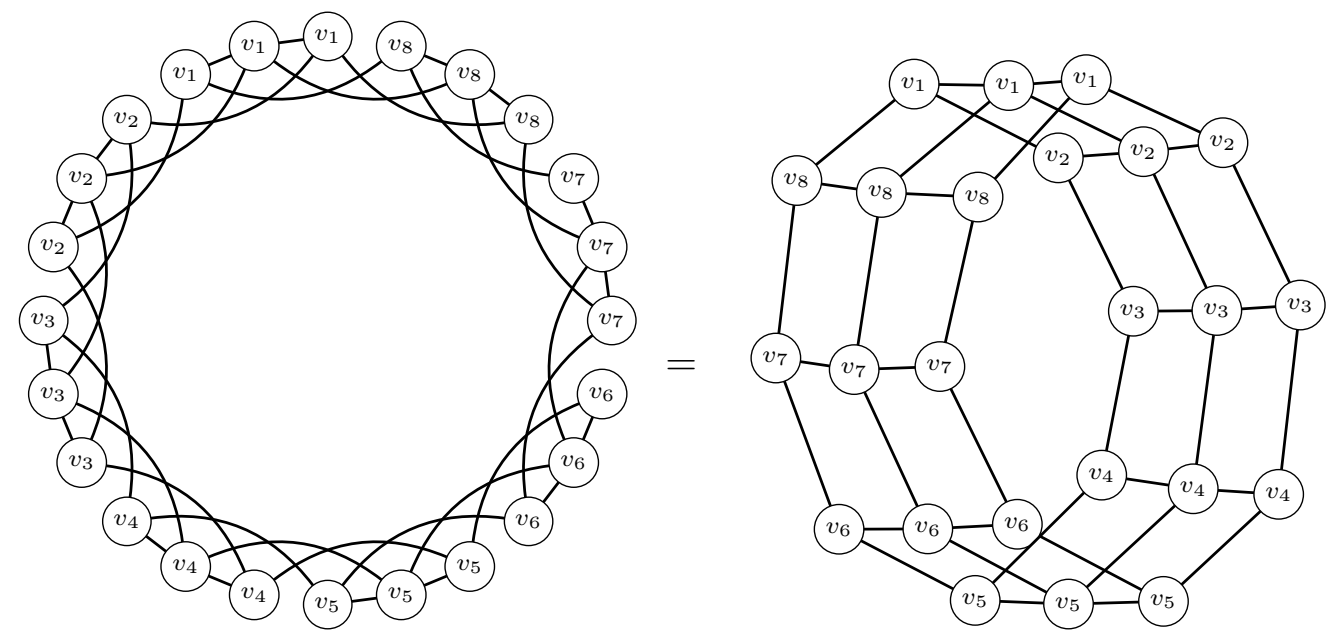

Figure 6: By applying edge deletions to $\operatorname{Circ}[24,\{1,3\}]$ and relabeling the vertices, it is clear that $C_{8} \square P_{3} \preceq$ $\operatorname{Circ}[24,\{1,3\}]$.

Proof. We will show that $Z=\{0,1, \ldots, 2 m-1\}$ is a zero forcing set. Suppose $s \in S$ and $s \neq m$. Then $1 \leq s<m$ and it follows that $m \pm s \in Z$. If $s=m$, then $m-s=m-m=0$ which implies $m-s \in Z$. This shows that all neighbors of $m$ except for $2 m$ are in $Z$; clearly $m \in Z$. Hence $m$ can force $2 m$. Using a similar argument $m+i$ forces $2 m+i$ for $i \in\{1,2, \ldots, n-2 m-1\}$. A forcing sequence is listed as

$$
m \rightarrow 2 m, m+1 \rightarrow 2 m+1, \ldots, m+(n-2 m-1)=n-m-1 \rightarrow 2 m+(n-2 m-1)=n-1 .
$$

Observation 3.14. Let $n$ be a multiple of $k, G=C_{n / k} \square P_{k}$, and $H=\operatorname{Circ}[n,\{1, k\}]$. Then $G \preceq H$. This is illustrated in Figure 6.

The next result may also be true for $n<24$, but our proof needs $n$ to be big enough to use results from $\mathrm{Z}\left(C_{8} \square P_{3}\right)=\mathrm{Z}(\operatorname{Circ}[24,\{1,3\}])=6$.

TheOrem 3.15. Let $n \geq 24$ be a multiple of 3 and let $G=\operatorname{Circ}[n,\{1,3\}]$. Then $\xi(G)=\mathrm{M}(G)=\mathrm{Z}(G)=$ 6.

Proof. In Example 3.1, we showed that $6=\xi\left(C_{8} \square P_{3}\right)$. By Observations 3.2 and $3.14 C_{8} \square P_{3} \preceq$ $C_{n / 3} \square P_{3} \preceq G$, and $\mathrm{Z}(G) \leq 6$ by Proposition 3.13. Therefore, $C_{8} \square P_{3}$ is a zero forcing minor of $G$, and $\xi(G)=\mathrm{M}(G)=\mathrm{Z}(G)=6$ by Theorem 3.5.

REMARK 3.16. For every positive integer $t, \operatorname{Circ}[2 t,\{1, t\}]$ is the Moebius ladder graph. The edges $\{i, i+t\}$ of Circ $[2 t,\{1, t\}]$ are the rungs in the Moebius ladder. It was shown in [3, Proposition 3.9] that all Moebius ladder graphs have both their maximum nullity and zero forcing number equal to 4 .

4. An application of vertex connectivity. In this section, we use known results for the vertex connectivity of a graph to show that the maximum nullity and zero forcing number for some circulant graphs are the same. 
305 Techniques for determining equality of the maximum nullity and the zero forcing number of a graph

The vertex connectivity, denoted by $\kappa(G)$, of a graph is the smallest number of vertices needed to be deleted to disconnect a noncomplete graph and $\kappa\left(K_{n}\right)=n-1$. In 2007, building on the work of Lovász, Saks, Schrijver [20], [19], Hein van der Holst [21] showed that the vertex connectivity of a graph is a lower bound for the maximum nullity of a graph. Although not published, it is worth noting that in a AIM workshop the minimum degree and vertex connectivity of a graph were used to show that the maximum nullity is equal to the zero forcing number for certain circulant graphs.

Theorem 4.1 ([21, Theorem 4]). Let $G$ be a graph. Then $\kappa(G) \leq \xi(G)$.

Corollary 4.2. Let $G$ be a graph. Then $\kappa(G) \leq \xi(G) \leq \mathrm{M}(G) \leq \mathrm{Z}(G)$.

Observation 4.3. Let $G$ be a circulant graph $\operatorname{Circ}[n, S]$ such that $S$ does not contain $\frac{n}{2}$. Then $\delta(G)=$ $2|S|$.

The circulant graph $\operatorname{Circ}[n,\{1,2, \ldots, t\}]$ is called a consecutive circulant. It is shown in [22, Theorem 4.1.5] that the vertex connectivity and the minimum degree of a consecutive circulant are equal.

Theorem 4.4 ([15, Corollary 2.2]). Let $2 t+1 \leq n$ and let $G=\operatorname{Circ}[n,\{1,2, \ldots, t\}]$. Then

$$
\kappa(G)=\delta(G)=\xi(G)=\mathrm{M}(G)=\mathrm{Z}(G)=2 t .
$$

Proof. By Observation 4.3, $\delta(G)=2 t$. Since $G$ is a consecutive circulant, $\kappa(G)=\delta(G)$. By Corollary 4.2, we have the following inequalities $\kappa(G)=\delta(G) \leq \xi(G) \leq \mathrm{M}(G) \leq \mathrm{Z}(G)$. An upper bound for the zero forcing number of $G$ is $2 t$, which is given by Proposition 3.13. Therefore, $\kappa(G)=\delta(G)=\xi(G)=\mathrm{M}(G)=\mathrm{Z}(G)=2 t$.

When $n$ is odd and $t=\left\lfloor\frac{n}{2}\right\rfloor$ the circulant $\operatorname{Circ}[n,\{1,2, \ldots, t\}]=K_{n}$. The equality of $\kappa, \delta, \xi$, and, Z shown for consecutive circulants in Theorem 4.4 is not true for all circulant graphs as shown in the next example.

ExAmple 4.5. Let $G$ be the graph $\operatorname{Circ}[8,\{1,3\}]=K_{4,4}$. By considering $G=K_{4,4}$, we see that $\kappa(G)=$ $\delta(G)=4$ and $\mathrm{Z}(G)=6$, since $\mathrm{Z}\left(K_{a, b}\right)=a+b-2$. It was shown in [6, Corollary 2.8] that $\xi(G)=$ $\min \{4,4\}+1=5$.

For $n=2 m+1$, if $n$ is prime, $\operatorname{gcd}(m-1, n)=\operatorname{gcd}(m, n)=1$. So $\operatorname{Circ}[n,\{1, \ldots, m-2, m\}] \cong$ $K_{n}-C_{n} \cong \operatorname{Circ}[n,[m-1]]$. However, $\operatorname{Circ}[22,\{1,2,3,4,5,6,7,8,10\}] \nRightarrow \operatorname{Circ}[22,\{1,2,3,4,5,6,7,8,9\}]$. Thus, the discussion below covers graphs that are not consecutive circulants.

Proposition 4.6. Let $H=\operatorname{Circ}[n,[m] \backslash\{m-1\}]$ where $n>9$ and $m=\lceil n / 2\rceil-1$. Then $\mathrm{Z}(H) \leq 2(m-1)$.

Proof. Observe first that $2(m-1)=\delta(H) \leq \mathrm{Z}(H)$. We first consider the case when $n$ is odd first. Then $n=2 m+1$. Since $m-1$ is not in the connection set, $i$ is not adjacent to $i+(m-1)$ or $i-(m-1)$. Note that $i-(m-1) \equiv i+n-(m-1) \equiv i+2 m+1-(m-1) \equiv i+m+2 \bmod n$. It follows that 0 is not adjacent to $m-1$ or $m+2$, and 3 is not adjacent to $m+2$ or $m+5$. Consider the set $Z=V(H) \backslash\{m-2, m-1, m+2\}$. Then $0 \rightarrow m-2$ and $3 \rightarrow m-1$. After these two forces, any vertex adjacent to $m+2$ can force $m+2$, which shows that $Z$ is a zero forcing set.

When $n$ is even, $n=2 m+2$. Since $m-1$ and $\frac{n}{2}$ are not in the connection set, $i$ is not adjacent to $i+(m-1), i-(m-1) \equiv i+m+3$ or $i+n / 2 \equiv i+(m+1)$. It follows that 0 is not adjacent to $m-1, m+1$, or $m+3$, and 2 is not adjacent $m+1, m+3$, or $m+5$. Consider the set $Z=V(H) \backslash\{2, m-1, m+3\}$. Then $0 \rightarrow 2$ and $2 \rightarrow m-1$. Any vertex adjacent to $m+3$ can force $m+3$, which shows that $Z$ is a zero forcing set. 
Theorem 4.7 ([9, Theorem 1]). Let $G$ be a circulant graph $\operatorname{Circ}\left[n,\left\{s_{1}, s_{2}, \ldots, s_{k}\right\}\right]$. There exists a proper divisor $d$ of $n$ such that the number of distinct positive residues modulo $d$ of $s_{1}, s_{2}, \ldots, s_{k}, n-s_{k}, n-$ $s_{k-1}, \ldots, n-s_{1}$ is less than $\min \left\{d-1, \frac{\delta(G)}{n} d\right\}$ if and only if $\kappa(G)<\delta(G)$.

Theorem 4.8. Let $H=\operatorname{Circ}[n,[m] \backslash\{m-1\}]$ where $n \geq 10$ and $m=\lceil n / 2\rceil-1$. Then $\kappa(G)=\delta(G)=$ $\xi(G)=\mathrm{M}(G)=\mathrm{Z}(G)=2(m-1)$.

Proof. Since $2(m-1)=\delta(G)=\mathrm{Z}(G)$, we need only to show $\kappa(G)=\delta(G)$. Let $d$ be a positive divisor of $n$ and let $S^{\prime}=\{1,2, \ldots, m-2, m, n-m, n-(m-2), \ldots, n-1\}$. If $d<m$, then $d-1 \leq m-2$ and $1,2, \ldots, d-1$ are $d-1$ distinct residue of $S^{\prime}$ modulo $d$. Note that $d=m$ is impossible since $m$ does not divide $2 m+1$ or $2 m+2$, as $m \geq 3$. If $n$ is even and $d=\frac{n}{2}$, then $\frac{\delta(G)}{n} d=\frac{\delta(G)}{2}=\frac{2(m-1)}{2}=m-1<d-1$. Furthermore $1,2, \ldots, m-2, m$ are $m-1$ distinct residue of $S^{\prime}$ modulo $d$ which is greater than or equal to $\frac{\delta(G)}{n} d$. Therefore, by Theorem 4.7 it must be the case that $\kappa(G)=\delta(G)$.

5. An application of equitable partitions. In this section, we use an equitable partition of a circulant graph to bound the nullity of the graph. It fact, the lower bound is obtained from the nullity of a circulant graph of small order which possesses the same connection set as the circulant graph of interest.

An equitable partition of a graph is a partition of the vertex set $V_{0}, V_{1}, \ldots, V_{k}$ such that for all $v \in V_{i}$ the number $b_{i j}$ of neighbors in $V_{j}$ is constant for all $V_{j}$. Let $V_{0}, V_{1}, \ldots, V_{k}$ be an equitable partition of $V(G)$. We say a divisor of $G$ is a weighted directed graph with vertex set $V_{0}, V_{1}, \ldots, V_{k}$ and $\operatorname{arc}\left(V_{i}, V_{j}\right)$ having weight $b_{i j}$ if and only if $b_{i j} \neq 0$. The matrix $\left[b_{i j}\right]$ is the divisor matrix associated with the equitable partition $V_{0}, V_{1}, \ldots, V_{k}$. It is known that an equitable partition of a graph $G$ can be used to find specific eigenvalues of $A(G)$ (see [10]).

EXAmple 5.1. Figure 7 shows the graph of the circulant Circ $[24,\{1,3\}]$. By partitioning the vertex set of Circ $[24,\{1,3\}]$ as in Figure 7, it is clear that the partition $V_{i}=\left\{i, i^{\prime}, i^{\prime \prime}\right\}$ for $i=0,1, \ldots, 7$ is an equitable partition of $\operatorname{Circ}[24,\{1,3\}]$.

Proposition 5.2 ([14, Page 196]). Let $\phi$ be an automorphism of $G$. Then the orbits of $\phi$ give an equitable partition of $V(G)$.
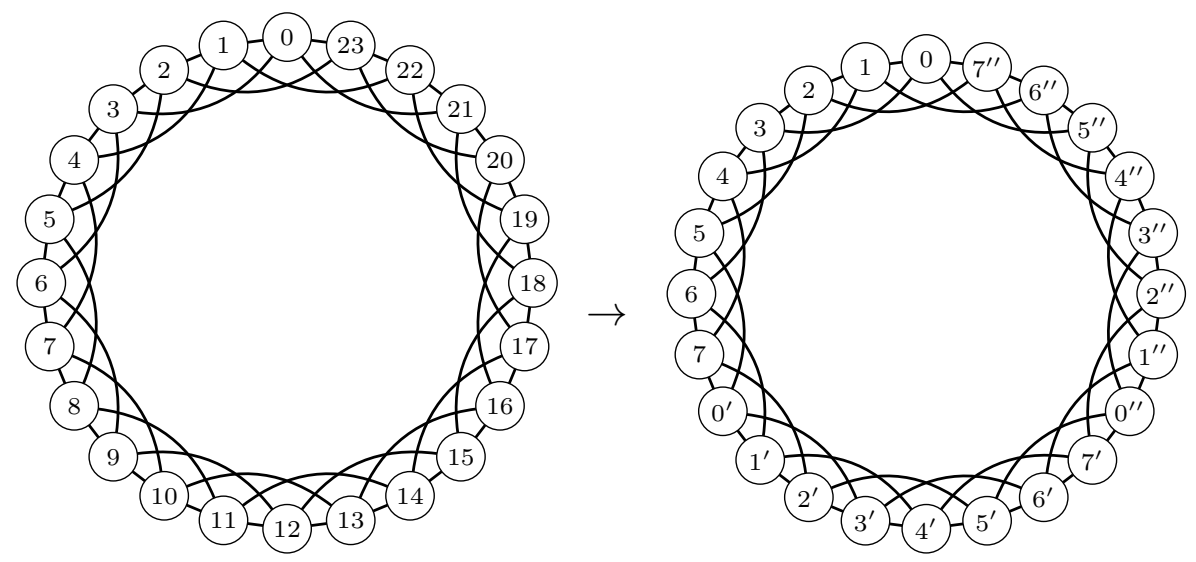

Figure 7: Circ $[24,\{1,3\}]$ and a relabeling showing how the vertices can be equitably partitioned. 
307 Techniques for determining equality of the maximum nullity and the zero forcing number of a graph

Note that the equitable partition in Example 5.1 is obtained from the automorphism $\varphi(i)=i+8$.

TheOrem 5.3 ([10, Theorem 3.9.5]). Let $G$ be a graph and let $D$ be a divisor matrix of some equitable partition of $V(G)$. Then the eigenvalues of $D$ are eigenvalues of $A(G)$ (including multiplicity).

TheOREM 5.4. Let $G$ be the circulant graph $\operatorname{Circ}[n k, S]$ where $k$ is a positive integer and $S \subseteq\left[\left\lceil\frac{n}{2}\right\rceil-1\right]$. Then the adjacency matrix of the circulant graph $\operatorname{Circ}[n, S]$ is a divisor matrix of $G$.

Proof. The orbits of the automorphism $\varphi(t) \equiv t+n \bmod n k$ of $G$ are

$$
V_{i}=\{r \in V(\operatorname{Circ}[n k, S]) \mid r \equiv i \bmod n\} .
$$

Hence, the partition $V_{0}, V_{1}, \ldots, V_{n-1}$ is an equitable partition of $G$.

Let $\left[b_{i j}\right]$ be the divisor matrix of $G$ with respect to the given equitable partition and let $\left[a_{i j}\right]$ be the adjacency matrix of $\operatorname{Circ}[n, S]$. It suffices to show for all $i$ and $j, b_{i j} \leq 1$ and $b_{i j}$ is nonzero if and only if $a_{i j}$ is nonzero. Suppose $s_{1}$ and $s_{2}$ are distinct elements in $S, V_{i} V_{j}$ is an arc, and $i+s_{1} \in V_{j}$. Since $s_{1}, s_{2} \in\left[\left\lceil\frac{n}{2}\right\rceil-1\right], s_{1} \pm s_{2} \not \equiv 0 \bmod n$ which implies $i+s_{1} \not \equiv i \pm s_{2} \bmod n$ and $i \pm s_{2} \not \equiv j \bmod n$. Hence $i \pm s_{2} \notin V_{j}$. Also $s_{1} \in\left[\left\lceil\frac{n}{2}\right\rceil-1\right]$, so $2 s_{1} \not \equiv 0 \bmod n$ which implies $i+s_{1} \not \equiv i-s_{1} \bmod n$ and $i-s_{1} \not \equiv j \bmod n$. This shows that $i-s_{1}, i+s_{2}, i-s_{2} \notin V_{j}$. Hence $b_{i j} \leq 1$ for all $i$ and $j$.

Suppose $V_{i}$ is adjacent to $V_{j}$. Then there exists a vertex $\ell \in V_{i}$ and $p \in V_{j}$ such that $\ell$ is adjacent to $p$, in $G$. Thus, $\ell-p \equiv i-j \bmod n$. By definition of adjacency in $G$, for some $s \in S, \ell \equiv p+s \bmod n k$ or $\ell \equiv p-s \bmod n k$. Hence $\ell-p \equiv s \bmod n k$ or $p-\ell \equiv s \bmod n k$. Thus, $i-j \equiv \ell-p \equiv s \bmod n$ or $i-j \equiv \ell-p \equiv-s \bmod n$. In either case, $i$ is adjacent to $j$ in $\operatorname{Circ}[n, S]$. Now suppose $i$ is adjacent to $j$ in $\operatorname{Circ}[n, S]$ where $0 \leq i, j \leq n-1$ as integers. Then it must be the case that $j=i+s \bmod n$ or $j=i-s \bmod n$. In $\operatorname{Circ}[n k, S], i \in V_{i}$ and $i+s \in V_{j}$ or $i-s \in V_{j}$. In either case, $b_{i j} \neq 0$ in the divisor matrix of $G$.

When $n$ is even in Theorem 5.4, the connection set cannot be extended to include $\frac{n}{2}$.

ExAmple 5.5. Let $G=\operatorname{Circ}[12,\{1,3\}]$ and $H=\operatorname{Circ}[6,\{1,3\}]=K_{3,3}$. Furthermore, using the equitable partition described in the proof of Theorem 5.4,

$$
V_{0}=\{0,6\}, V_{1}=\{1,7\}, V_{2}=\{2,8\}, V_{3}=\{3,9\}
$$

$b_{0,1}=1, b_{0,2}=0, b_{0,3}=2$,

$$
\left[b_{i j}\right]=\left(\begin{array}{cccccc}
0 & 1 & 0 & 2 & 0 & 1 \\
1 & 0 & 1 & 0 & 2 & 0 \\
0 & 1 & 0 & 1 & 0 & 2 \\
2 & 0 & 1 & 0 & 1 & 0 \\
0 & 2 & 0 & 1 & 0 & 1 \\
1 & 0 & 2 & 0 & 1 & 0
\end{array}\right)
$$

and $A(\operatorname{Circ}[6,\{1,3\}])$ is not the divisor matrix of $G$. By computation, the eigenvalues of $A(G)$ are

$$
\pm 4, \pm \sqrt{3}, \pm 1,0
$$

and $H$ is bipartite and 3 - regular which implies \pm 3 are eigenvalues of $H$. This shows that the adjacency matrix of $H$ is not a divisor matrix of $G$.

The next corollary is a direct result of Theorems 5.3 and 5.4. 
Corollary 5.6. Consider the circulant graph $\operatorname{Circ}[n k, S]$ where $k$ is a positive integer and $S \subseteq\left[\left\lceil\frac{n}{2}\right\rceil-1\right]$. Then

$$
\operatorname{spec}(A(\operatorname{Circ}[n, S])) \subseteq \operatorname{spec}(A(\operatorname{Circ}[n k, S])),
$$

and $\operatorname{null}(A(\operatorname{Circ}[n, S])) \leq \operatorname{null}(A(\operatorname{Circ}[n k, S]))$.

It was shown in Theorem 3.15 that $\mathrm{M}(\operatorname{Circ}[3 k,\{1,3\}])=\mathrm{Z}(\operatorname{Circ}[3 k,\{1,3\}])=6$ for $k \geq 8$. The next result establishes field independence, in addition to showing that the maximum nullity equals the zero forcing number for many additional circulants..

THEOREM 5.7. Let $k$ be a positive integer and let $\ell$ be an odd integer between 3 and 21. Then

$$
\mathrm{M}\left(\operatorname{Circ}\left[\left(\ell^{2}-1\right) k,\{1, \ell\}\right]\right)=\mathrm{Z}\left(\operatorname{Circ}\left[\left(\ell^{2}-1\right) k,\{1, \ell\}\right]\right)=2 \ell,
$$

$\operatorname{Circ}\left[\left(\ell^{2}-1\right) k,\{1, \ell\}\right]$ has field independent minimum rank, and its adjacency matrix is an universally optimal matrix.

Proof. Let $n=\ell^{2}-1, S=\{1, \ell\}$, and $G=\operatorname{Circ}[n k, S]$ for $k \geq 1$. By Proposition 3.13 and Proposition $1.1, \mathrm{M}(G) \leq \mathrm{Z}(G) \leq 2 \ell$. Thus, it suffices to show that $\operatorname{null}(A(\operatorname{Circ}[n, S]))=2 \ell$. This is easily verified using computer software. (SageMath offers commands for computing the adjacency matrix of a graph and its nullity.)

Conjecture 5.8. For all positive values of $k$ and odd $\ell$,

$$
\mathrm{M}\left(\operatorname{Circ}\left[\left(\ell^{2}-1\right) k,\{1, \ell\}\right]\right)=\mathrm{Z}\left(\operatorname{Circ}\left[\left(\ell^{2}-1\right) k,\{1, \ell\}\right]\right)=2 \ell,
$$

and field independent minimum rank with universally optimal matrix $A(G)$.

6. An application of equitable decompositions. In this section, we use the equitable decomposition, introduced in [7], of the adjacency matrix to establish field independent minimum rank of a graph. The graphs of interest are the extended cube graphs ECG $(6 q+1,6 q+1)$ where $q$ is a nonnegative integer.

An automorphism of a graph $G$ is an isomorphism $\phi$ from $V(G)$ to $V(G)$ such that $\phi(i)$ is adjacent to $\phi(j)$ if and only if $i$ is adjacent to $j$. Let $G$ be a graph with $v, u \in V(G)$ and let $\phi$ be an automorphism of $G$. Define the relation $\approx$ on the vertices of $G$ by $v \approx u$ if and only if there exists a nonnegative integer $j$ for which $v=\phi^{j}(u)$. This relation is an equivalence relation on the vertices of $G$ and the equivalence classes are the orbits of $\phi$. Let $\phi$ be an uniform automorphism of $G$ with orbit size $k$ where $1<k$. A transversal of $\phi$ is a subset of $V(G)$ containing exactly one vertex from each orbit of $\phi$. The $\ell$-power of transversal $T$ is defined to be the following transversal:

$$
T_{\ell}=\left\{\phi^{\ell}(v) \mid v \in T\right\},
$$

for $\ell \in\{0,1,2, \ldots, k-1\}$. It is straightforward to see that $T_{\ell}$ is a transversal and $\cup_{\ell=0}^{k-1} T_{\ell}=V(G)$.

Given an automorphism $\phi$, an $n \times n$ matrix $A=\left[a_{i j}\right]$ associated with the graph $G$ on $n$ vertices such that

$$
a_{\phi(i), \phi(j)}=a_{i j},
$$

for all $i, j \in\{1,2, \ldots, n\}$, is called $\phi$-compatible. An $n \times n$ matrix $A$ associated with the graph $G$ is called $\phi$-automorphism compatible if it is $\phi$-compatible for every automorphism $\phi$ of $G$. Recently in 2017, Barrett et al. used equitable partitions of a graph in [7] to decompose $A(G)$. This decomposition can be used to determine all eigenvalues of $A(G)$. As a result, this decomposition is useful for determining a lower bound for the maximum nullity. Moreover, it can be used to establish a potential candidate for an universally optimal matrix. 
309 Techniques for determining equality of the maximum nullity and the zero forcing number of a graph

EXAMPLE 6.1. In general, the extended cube graphs do not have field independent minimum rank. Some extended cube graphs are isomorphic to the Cartesian product of a cycle and a path. For instance, ECG(0,3) is isomorphic to $C_{7} \square P_{2}$. It was shown in Example 1.8 that $\operatorname{mr}\left(\mathbb{Z}_{2}, C_{7} \square P_{2}\right) \neq \operatorname{mr}\left(C_{7} \square P_{2}\right)$.

OBSERVATION 6.2. The adjacency matrix of a graph is automorphism compatible.

The next theorem is stated in [7] for automorphism compatible matrices, but as noted there it could be stated for a $\phi$-compatible matrix and we do so.

TheOREM 6.3 ([7, Theorem 3.8]). Let $G$ be a graph on $n$ vertices, let $\phi$ be an uniform automorphism of $G$ of orbit size $k$, let $\mathrm{T}_{0}$ be a transversal of the orbits of $\phi$, and let $A$ be an $\phi$-compatible matrix in $\mathcal{S}(G)$. Set $A_{\ell}=A\left[\mathrm{~T}_{0}, \mathrm{~T}_{\ell}\right], \ell=0,1, \ldots, k-1$, let $\omega=e^{2 \pi i / k}$, and define

$$
B_{j}=\sum_{\ell=0}^{k-1} \omega^{j \ell} A_{\ell}, \quad j=0,1, \ldots, k-1 .
$$

Then for some invertible matrix $S$

$$
S^{-1} A S=B_{0} \oplus B_{1} \oplus \cdots \oplus B_{k-1}
$$

and

$$
\sigma(A)=\sigma\left(B_{0}\right) \cup \sigma\left(B_{1}\right) \cup \cdots \cup \sigma\left(B_{k-1}\right) .
$$

The decomposition in (3) is called an equitable decomposition of $A$.

OBservation 6.4. Let $G$ be an extended cube graph $\operatorname{ECG}(t, t)$ on $n$ vertices and let $r=\frac{n}{4}$. Then the function $\varphi(x) \equiv x+r \bmod n$ is a uniform automorphism for $G$. The function $\varphi$ can also be written as a permutation,

$$
\phi=(0,0+r, 0+2 r, 0+3 r)(1,1+r, 1+2 r, 1+3 r) \cdots(r-1, r-1+r, r-1+2 r, r-1+3 r) .
$$

Furthermore, $T_{0}=\{0,1, \ldots, r-1\}$ is a transversal.

ExAmPLE 6.5. The following is an example of constructing the eigenvalues of ECG(1,1) using an equitable decomposition. As in Observation 6.4,

$$
\varphi(x) \equiv x+3 \bmod 12
$$

is an automorphism with permutation representation $\phi=(0,3,6,9)(1,4,7,10)(2,5,8,11)$, and the transversals are $T_{0}=\{0,1,2\}, T_{1}=\{3,4,5\}, T_{2}=\{6,7,8\}, T_{3}=\{9,10,11\}$. Let

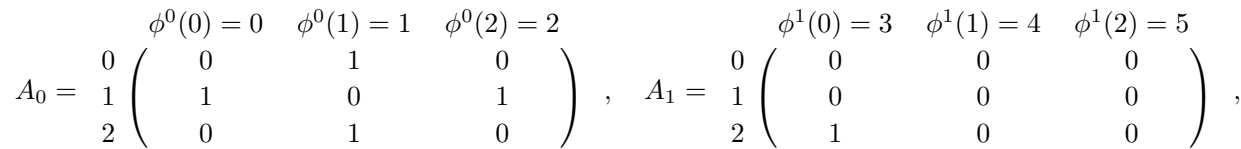

$$
\begin{aligned}
& \begin{array}{ccc}
\phi^{2}(0)=6 & \phi^{2}(1)=7 & \phi^{2}(2)=8 \\
A_{2}= & = \\
2
\end{array}\left(\begin{array}{ccc}
0 & 0 & 1 \\
0 & 1 & 0 \\
1 & 0 & 0
\end{array}\right), \quad \text { and } \quad A_{3}=\begin{array}{c}
0 \\
\phi^{3}(0)=9
\end{array}\left(\begin{array}{ccc}
0 & \phi^{3}(1)=10 & \phi^{3}(2)=11 \\
0 & 0 & 1 \\
0 & 0 & 0 \\
0
\end{array}\right) .
\end{aligned}
$$


Hence

$$
B_{0}=A_{0}+A_{1}+A_{2}+A_{3}=\left(\begin{array}{ccc}
0 & 1 & 2 \\
1 & 1 & 1 \\
2 & 1 & 0
\end{array}\right)
$$

and it follows that the spectrum of $B_{0}$ is $\{3,0,-2\}$ with eigenvector $x_{0}=[1,-2,1]^{T}$ corresponding to the eigenvalue 0. Also,

$$
B_{1}=A_{0}+i A_{1}-A_{2}-i A_{3}=\left(\begin{array}{ccc}
0 & 1 & -1-i \\
1 & -1 & 1 \\
-1+i & 1 & 0
\end{array}\right)
$$

and the spectrum of $B_{1}$ is approximately $\{1.561552,0,-2.561552\}$ with eigenvector $x_{1}=[i, 1+i, 1]^{T}$ corresponding to the eigenvalue 0 ,

$$
B_{2}=A_{0}-A_{1}+A_{2}-A_{3}=\left(\begin{array}{ccc}
0 & 1 & 0 \\
1 & 1 & 1 \\
0 & 1 & 0
\end{array}\right)
$$

has spectrum $\{2,0,-1\}$ with eigenvector $x_{2}=[1,0,-1]^{T}$ corresponding to the eigenvalue 0 , and

$$
B_{3}=A_{0}-i A_{1}-A_{2}+i A_{3}=\left(\begin{array}{ccc}
0 & 1 & -1+i \\
1 & 1 & 1 \\
-1-i & 1 & 0
\end{array}\right),
$$

has spectrum approximately $\{1.561552,0,-2.561552\}$ with eigenvector $x_{3}=[-1,-1-i, i]^{T}$ corresponding to the eigenvalue 0. Using SageMath (see [23]), we compute the eigenvalues of ECG(1,1) to be approximately

$$
\{3,2,1.561552,1.561552,0,0,0,0,-1,-2,-2.561552,-2.561552\}
$$

which is the union of the spectra of $B_{0}, B_{1}, B_{2}, B_{3}$.

THEOREM 6.6. Let $G$ be an extended cube graph $\operatorname{ECG}(6 q+1,6 q+1)$ for some nonnegative integer $q$. Then $G$ has field independent minimum rank and $A(G)$ is a universally optimal matrix.

Proof. First we will show that the adjacency matrix of each such extended cube graph has nullity at least 4 . Hence by Corollary 3.11 the adjacency matrix realizes the maximum nullity.

It was shown in Example 6.5 that the nullity of $\operatorname{ECG}(1,1)$ has nullity equal to 4 , so we assume $q>0$. Let $G$ be an extended cube graph $\operatorname{ECG}(6 q+1,6 q+1)$ and let $n$ be the number of vertices of $G$. Consider the uniform automorphism

$$
\varphi(x)=x+r \bmod n,
$$

where $r=\frac{n}{4}$ given by Observation 6.4. By Theorem 6.3, $G$ has the following spectrum:

$$
\operatorname{spec}(A(G))=\operatorname{spec}\left(B_{0}\right) \cup \operatorname{spec}\left(B_{1}\right) \cup \operatorname{spec}\left(B_{2}\right) \cup \operatorname{spec}\left(B_{3}\right),
$$

for the matrices $B_{i}$ corresponding to $\varphi$. We show that $B_{0}, B_{1}, B_{2}, B_{3}$ each have nullity at least 1 , which implies $A(G)$ has nullity at least 4 .

The transversals with respect to $\varphi$ are $T_{0}=\{0,1,2, \ldots, r-1\}, T_{1}=\{r, r+1, r+2, \ldots, 2 r-1\}$, $T_{2}=\{2 r, 2 r+1,2 r+2, \ldots, 3 r-1\}, T_{3}=\{3 r, 3 r+1,3 r+2, \ldots, 4 r-1\}$. Hence $k=4$ and $\omega=e^{2 \pi i / 4}=i$. 
311 Techniques for determining equality of the maximum nullity and the zero forcing number of a graph

For the graph $\operatorname{ECG}(1,1)$, let $\tilde{A}_{0}, \tilde{A}_{1}, \tilde{A}_{2}, \tilde{A}_{3}$ be the corresponding matrices used in Theorem 6.3 to construct $\tilde{B}_{0}, \tilde{B}_{1}, \tilde{B}_{2}, \tilde{B}_{3}$ such that

$$
\operatorname{spec}(A(\operatorname{ECG}(1,1)))=\operatorname{spec}\left(\tilde{B}_{0}\right) \cup \operatorname{spec}\left(\tilde{B}_{1}\right) \cup \operatorname{spec}\left(\tilde{B}_{2}\right) \cup \operatorname{spec}\left(\tilde{B}_{3}\right),
$$

and $\tilde{B}_{0}=\tilde{A}_{0}+\tilde{A}_{1}+\tilde{A}_{2}+\tilde{A}_{3}$. Also, let $\tilde{x}_{0}, \tilde{x}_{1}, \tilde{x}_{2}$ be the eigenvectors of $\tilde{B}_{0}, \tilde{B}_{1}, \tilde{B}_{2}$, respectively, corresponding to the eigenvalue 0 . It follows that $A_{0}, A_{1}, A_{2}, A_{3}$ are the matrices

$$
\begin{aligned}
& A_{0}=\left(\begin{array}{ccccccc}
\tilde{A}_{0} & \tilde{A}_{1} & 0 & 0 & 0 & \cdots & 0 \\
\tilde{A}_{3} & \tilde{A}_{0} & \tilde{A}_{1} & 0 & 0 & \cdots & 0 \\
0 & \tilde{A}_{3} & \tilde{A}_{0} & \tilde{A}_{1} & 0 & \cdots & 0 \\
\vdots & \vdots & \ddots & \ddots & \ddots & \vdots & \vdots \\
0 & \cdots & 0 & \tilde{A}_{3} & \tilde{A}_{0} & \tilde{A}_{1} & 0 \\
0 & \cdots & 0 & 0 & \tilde{A}_{3} & \tilde{A}_{0} & \tilde{A}_{1} \\
0 & \cdots & 0 & 0 & 0 & \tilde{A}_{3} & \tilde{A}_{0}
\end{array}\right) \\
& A_{1}=\left(\begin{array}{ccccccc}
0 & 0 & 0 & 0 & 0 & \cdots & 0 \\
0 & 0 & 0 & 0 & 0 & \cdots & 0 \\
0 & 0 & 0 & 0 & 0 & \cdots & 0 \\
\vdots & \vdots & \vdots & \ddots & \vdots & \vdots & \vdots \\
0 & 0 & 0 & 0 & 0 & \cdots & 0 \\
0 & 0 & 0 & 0 & 0 & \cdots & 0 \\
\tilde{A}_{1} & 0 & 0 & 0 & 0 & \cdots & 0
\end{array}\right) \\
& A_{2}=\left(\begin{array}{ccccccc}
0 & 0 & 0 & \cdots & 0 & 0 & \tilde{A}_{2} \\
0 & 0 & 0 & \cdots & 0 & \tilde{A}_{2} & 0 \\
0 & 0 & 0 & \cdots & \tilde{A}_{2} & 0 & 0 \\
\vdots & \vdots & \vdots & . & \vdots & \vdots & \vdots \\
0 & 0 & \tilde{A}_{2} & \cdots & 0 & 0 & 0 \\
0 & \tilde{A}_{2} & 0 & \cdots & 0 & 0 & 0 \\
\tilde{A}_{2} & 0 & 0 & \cdots & 0 & 0 & 0
\end{array}\right), \quad \text { and } \quad A_{3}=A_{1}^{T}
\end{aligned}
$$

By definition,

$$
B_{j}=i^{0 j} A_{0}+i^{j} A_{1}+i^{2 j} A_{2}+i^{3 j} A_{3}=A_{0}+i^{j} A_{1}+(-1)^{j} A_{2}+i^{3 j} A_{3}
$$

for $j=0,1,2,3$, so the following matrices are constructed:

$$
\begin{array}{ll}
B_{0}=A_{0}+A_{1}+A_{2}+A_{3} & B_{1}=A_{0}+i A_{1}-A_{2}-i A_{3} \\
B_{2}=A_{0}-A_{1}+A_{2}-A_{3} & B_{3}=A_{0}-i A_{1}-A_{2}+i A_{3} .
\end{array}
$$


Writing $B_{j}$ in terms of the matrices $\tilde{A}_{0}, \tilde{A}_{1}, \tilde{A}_{2}, \tilde{A}_{3}$, we get the following matrix

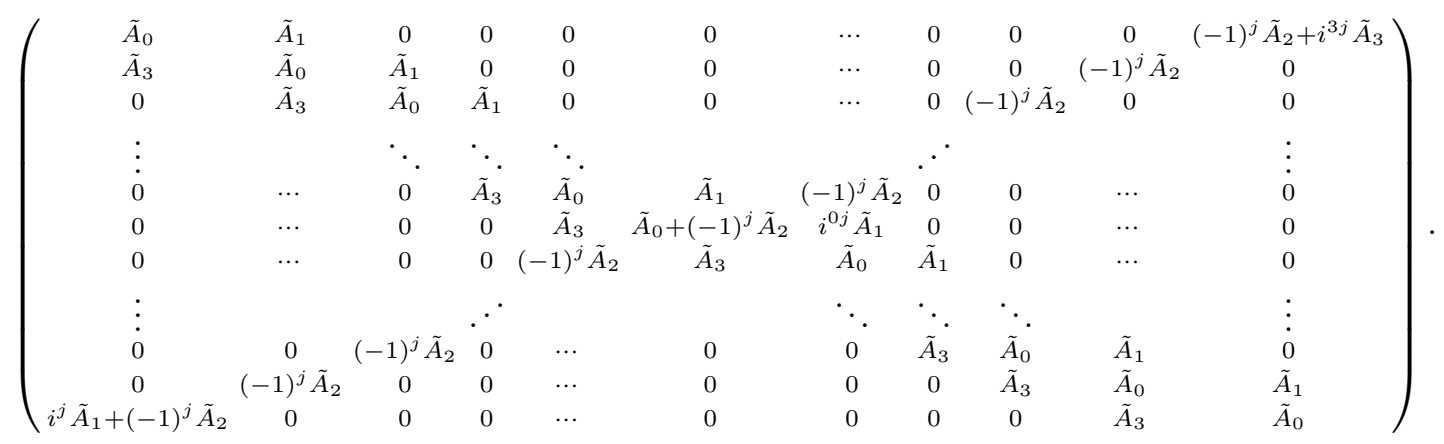

For simplicity of notation let $\hat{x}_{1}=-\tilde{A}_{2} \tilde{x}_{1}$ and $\hat{x}_{2}=-\tilde{x}_{2}$. We show that $x_{0}=\bigoplus_{m=1}^{2 q+1} \tilde{x}_{0}, x_{1}=$ $\bigoplus_{m=1}^{q}\left(\tilde{x}_{1} \oplus \hat{x}_{1}\right) \oplus \tilde{x}_{1}$, and $x_{2}=\bigoplus_{m=1}^{q}\left(\tilde{x}_{2} \oplus \hat{x}_{2}\right) \oplus \tilde{x}_{2}$ are eigenvectors corresponding to eigenvalue 0 for $B_{0}$, $B_{1}$, and $B_{2}$, respectively. Since $B_{3}=B_{1}^{T}$ it follows that $B_{3}$ also has a zero eigenvalue so we omit showing that $B_{3}$ has an eigenvalue of zero. Observe that

$$
B_{0}=\left(\begin{array}{ccccccccccc}
\tilde{A}_{0} & \tilde{A}_{1} & 0 & 0 & 0 & 0 & \cdots & 0 & 0 & 0 & \tilde{A}_{2}+\tilde{A}_{3} \\
\tilde{A}_{3} & \tilde{A}_{0} & \tilde{A}_{1} & 0 & 0 & 0 & \cdots & 0 & 0 & \tilde{A}_{2} & 0 \\
0 & \tilde{A}_{3} & \tilde{A}_{0} & \tilde{A}_{1} & 0 & 0 & \cdots & 0 & \tilde{A}_{2} & 0 & 0 \\
\vdots & & \ddots & \ddots & \ddots & & & . & & & \vdots \\
0 & \cdots & 0 & \tilde{A}_{3} & \tilde{A}_{0} & \tilde{A}_{1} & \tilde{A}_{2} & 0 & 0 & \cdots & 0 \\
0 & \cdots & 0 & 0 & \tilde{A}_{3} & \tilde{A}_{0}+\tilde{A}_{2} & \tilde{A}_{1} & 0 & 0 & \cdots & 0 \\
0 & \cdots & 0 & 0 & \tilde{A}_{2} & \tilde{A}_{3} & \tilde{A}_{0} & \tilde{A}_{1} & 0 & \cdots & 0 \\
\vdots & & & . \cdot & & & \ddots & \ddots & \ddots & & \vdots \\
0 & 0 & \tilde{A}_{2} & 0 & \cdots & 0 & 0 & \tilde{A}_{3} & \tilde{A}_{0} & \tilde{A}_{1} & 0 \\
0 & \tilde{A}_{2} & 0 & 0 & \cdots & 0 & 0 & 0 & \tilde{A}_{3} & \tilde{A}_{0} & \tilde{A}_{1} \\
\tilde{A}_{1}+\tilde{A}_{2} & 0 & 0 & 0 & \cdots & 0 & 0 & 0 & 0 & \tilde{A}_{3} & \tilde{A}_{0}
\end{array}\right) .
$$

The product $B_{0} x_{0}$ reduces down to the following vector:

$$
\left(\begin{array}{c}
\tilde{A}_{0} \tilde{x}_{0}+\tilde{A}_{1} \tilde{x}_{0}+\tilde{A}_{2} \tilde{x}_{0}+\tilde{A}_{3} \tilde{x}_{0} \\
\tilde{A}_{0} \tilde{x}_{0}+\tilde{A}_{1} \tilde{x}_{0}+\tilde{A}_{2} \tilde{x}_{0}+\tilde{A}_{3} \tilde{x}_{0} \\
\vdots \\
\tilde{A}_{0} \tilde{x}_{0}+\tilde{A}_{1} \tilde{x}_{0}+\tilde{A}_{2} \tilde{x}_{0}+\tilde{A}_{3} \tilde{x}_{0} \\
\tilde{A}_{0} \tilde{x}_{0}+\tilde{A}_{1} \tilde{x}_{0}+\tilde{A}_{2} \tilde{x}_{0}+\tilde{A}_{3} \tilde{x}_{0}
\end{array}\right)=\left(\begin{array}{c}
\tilde{B}_{0} \tilde{x}_{0} \\
\tilde{B}_{0} \tilde{x}_{0} \\
\vdots \\
\tilde{B}_{0} \tilde{x}_{0} \\
\tilde{B}_{0} \tilde{x}_{0}
\end{array}\right)=0,
$$

since $\tilde{B}_{0}=\tilde{A}_{0}+\tilde{A}_{1}+\tilde{A}_{2}+\tilde{A}_{3}$.

To compute $B_{1} x_{1}$, consider the fact that $\tilde{x}_{1}=[i, 1+i, 1]^{T}$ is an eigenvector for $\tilde{B}_{1}$. So by definition, $\hat{x}_{1}=[-1,-1-i,-i]^{T}$ and

$$
\begin{gathered}
\tilde{A}_{0} \hat{x}_{1}=\left(\begin{array}{lll}
0 & 1 & 0 \\
1 & 0 & 1 \\
0 & 1 & 0
\end{array}\right)\left(\begin{array}{c}
-1 \\
-1-i \\
-i
\end{array}\right)=-A_{0} \tilde{x}_{1} \\
\tilde{A}_{1} \hat{x}_{1}=\left(\begin{array}{lll}
0 & 0 & 0 \\
0 & 0 & 0 \\
1 & 0 & 0
\end{array}\right)\left(\begin{array}{c}
-1 \\
-1-i \\
-i
\end{array}\right)=\left(\begin{array}{c}
0 \\
0 \\
-1
\end{array}\right)=i\left(\begin{array}{lll}
0 & 0 & 0 \\
0 & 0 & 0 \\
1 & 0 & 0
\end{array}\right)\left(\begin{array}{c}
i \\
1+i \\
1
\end{array}\right)=i \tilde{A}_{1} \tilde{x}_{1} .
\end{gathered}
$$


313 Techniques for determining equality of the maximum nullity and the zero forcing number of a graph

Since $\tilde{A}_{2}^{2}=I$ it follows that $\tilde{A}_{2} \hat{x}_{1}=-\tilde{x}_{1}$. Also,

$$
\tilde{A}_{3} \hat{x}_{1}=\left(\begin{array}{lll}
0 & 0 & 1 \\
0 & 0 & 0 \\
0 & 0 & 0
\end{array}\right)\left(\begin{array}{c}
-1 \\
-1-i \\
-i
\end{array}\right)=\left(\begin{array}{c}
-i \\
0 \\
0
\end{array}\right)=-i\left(\begin{array}{lll}
0 & 0 & 1 \\
0 & 0 & 0 \\
0 & 0 & 0
\end{array}\right)\left(\begin{array}{c}
i \\
1+i \\
1
\end{array}\right)=-i \tilde{A}_{3} \tilde{x}_{1}
$$

In other words,

$$
\tilde{A}_{0} \hat{x}_{1}=(-1-i) \mathbf{1}^{T}, \tilde{A}_{1} \hat{x}_{1}=i \tilde{A}_{1} \tilde{x}_{1}, \tilde{A}_{2} \hat{x}_{1}=-\tilde{x}_{1} \text {, and } \tilde{A}_{3} \hat{x}_{1}=-i \tilde{A}_{3} \tilde{x}_{1} \text {, }
$$

and these values are used to reduce the entries of the next product. We have that $B_{1} x_{1}$ is

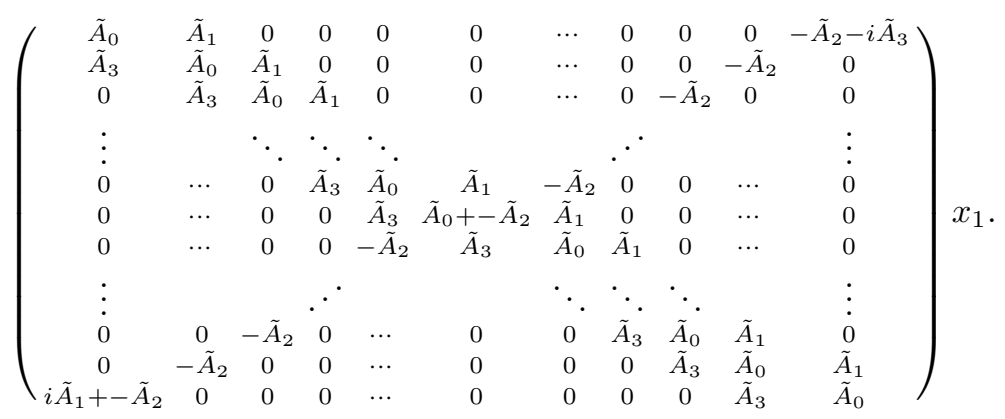

We show that the product $B_{1} x_{1}$ is given by the vector,

$$
\left(\begin{array}{c}
\tilde{A}_{0} \tilde{x}_{1}+\tilde{A}_{1} \hat{x}_{1}+\left(-\tilde{A}_{1}-i \tilde{A}_{3}\right) \tilde{x}_{1} \\
-------------- \\
\tilde{A}_{3} \tilde{x}_{1}+\tilde{A}_{0} \hat{x}_{1}+\tilde{A}_{1} \tilde{x}_{1}+\left(-\tilde{A}_{2} \hat{x}_{1}\right) \\
\tilde{A}_{3} \hat{x}_{1}+\tilde{A}_{0} \tilde{x}_{1}+\tilde{A}_{1} \hat{x}_{1}+\left(-\tilde{A}_{2} \tilde{x}_{1}\right) \\
\tilde{A}_{3} \tilde{x}_{1}+\tilde{A}_{0} \hat{x}_{1}+\tilde{A}_{1} \tilde{x}_{1}+\left(-\tilde{A}_{2} \hat{x}_{1}\right) \\
\tilde{A}_{3} \hat{x}_{1}+\tilde{A}_{0} \tilde{x}_{1}+\tilde{A}_{1} \hat{x}_{1}+\left(-\tilde{A}_{2} \tilde{x}_{1}\right) \\
\vdots \\
\tilde{A}_{3} \hat{x}_{1}+\tilde{A}_{0} \tilde{x}_{1}+\tilde{A}_{1} \hat{x}_{1}+\left(-\tilde{A}_{2} \tilde{x}_{1}\right) \\
\tilde{A}_{3} \tilde{x}_{1}+\tilde{A}_{0} \hat{x}_{1}+\tilde{A}_{1} \tilde{x}_{1}+\left(-\tilde{A}_{2} \hat{x}_{1}\right) \\
\left.------------------\tilde{A}_{1}\right) \tilde{x}_{1}+\tilde{A}_{1} \hat{x}_{1} \\
\tilde{A}_{3} \hat{x}_{1}+\left(\tilde{A}_{0}-\tilde{A}_{2} \tilde{A}_{2}\right. \\
\left.------------\tilde{A}_{2} \tilde{x}_{1}\right) \\
\tilde{A}_{3} \hat{x}_{1}+\tilde{A}_{0} \tilde{x}_{1}+\tilde{A}_{1} \hat{x}_{1}+\left(-\tilde{x}_{1}\right) \\
\tilde{A}_{3} \tilde{x}_{1}+\tilde{A}_{0} \hat{x}_{1}+\tilde{A}_{1} \tilde{x}_{1}+\left(-\tilde{A}_{2} \hat{x}_{1}\right) \\
\vdots \\
\tilde{A}_{3} \tilde{x}_{1}+\tilde{A}_{0} \hat{x}_{1}+\tilde{A}_{1} \tilde{x}_{1}+\left(-\tilde{A}_{2} \hat{x}_{1}\right) \\
\tilde{A}_{3} \hat{x}_{1}+\tilde{A}_{0} \tilde{x}_{1}+\tilde{A}_{1} \hat{x}_{1}+\left(-\tilde{A}_{2} \tilde{x}_{1}\right) \\
--------------- \\
\left(i \tilde{A}_{1}-\tilde{A}_{2}\right) \tilde{x}_{1}+\tilde{A}_{3} \hat{x}_{1}+\tilde{A}_{0} \tilde{x}_{1}
\end{array}\right)=0
$$


which implies that both matrices $B_{1}$ and $B_{3}$ have a zero eigenvalue. Note that each entry in the product takes on one of the following values:

$$
\begin{aligned}
\tilde{A}_{0} \tilde{x}_{1}+\tilde{A}_{1} \hat{x}_{1}+\left(-\tilde{A}_{2}-i \tilde{A}_{3}\right) \tilde{x}_{1}= & \tilde{A}_{0} \tilde{x}_{1}+i \tilde{A}_{1} \tilde{x}_{1}-\tilde{A}_{2} \tilde{x}_{1}-i \tilde{A}_{3} \tilde{x}_{1} \\
= & \left(\tilde{A}_{0}+i \tilde{A}_{1}-\tilde{A}_{2}-i \tilde{A}_{3}\right) \tilde{x}_{1}=\tilde{B}_{1} \tilde{x}_{1}=0 \\
\tilde{A}_{3} \tilde{x}_{1}+\tilde{A}_{0} \hat{x}_{1}+\tilde{A}_{1} \tilde{x}_{1}+\left(-\tilde{A}_{2} \hat{x}_{1}\right)= & (1,0,0)^{T}+(-1-i,-1-i,-1-i)^{T} \\
& +(0,0, i)^{T}+(i, 1+i, 1)^{T}=0 \\
\tilde{A}_{3} \hat{x}_{1}+\tilde{A}_{0} \tilde{x}_{1}+\tilde{A}_{1} \hat{x}_{1}+\left(-\tilde{A}_{2} \tilde{x}_{1}\right)= & -i \tilde{A}_{3} \tilde{x}_{1}+\tilde{A}_{0} \tilde{x}_{1}+i \tilde{A}_{1} \tilde{x}_{1}-\tilde{A}_{2} \tilde{x}_{1} \\
= & \left(\tilde{A}_{0}+i \tilde{A}_{1}-\tilde{A}_{2}-i \tilde{A}_{3}\right) \tilde{x}_{1}=\tilde{B}_{1} \tilde{x}_{1}=0 \\
\tilde{A}_{3} \hat{x}_{1}+\left(\tilde{A}_{0}-\tilde{A}_{2}\right) \tilde{x}_{1}+\tilde{A}_{1} \hat{x}_{1}= & 0 \quad \text { by }(4) \\
\left(i \tilde{A}_{1}-\tilde{A}_{2}\right) \tilde{x}_{1}+\tilde{A}_{3} \hat{x}_{1}+\tilde{A}_{0} \tilde{x}_{1}= & i \tilde{A}_{1} \tilde{x}_{1}-\tilde{A}_{2} \tilde{x}_{1}-i \tilde{A}_{3} \tilde{x}_{1}+\tilde{A}_{0} \tilde{x}_{1} \\
= & \left(\tilde{A}_{0}+i \tilde{A}_{1}-\tilde{A}_{2}-i \tilde{A}_{3}\right) \tilde{x}_{1}=\tilde{B}_{1} \tilde{x}_{1}=0 .
\end{aligned}
$$

Finally, we compute $B_{2} x_{2}$,

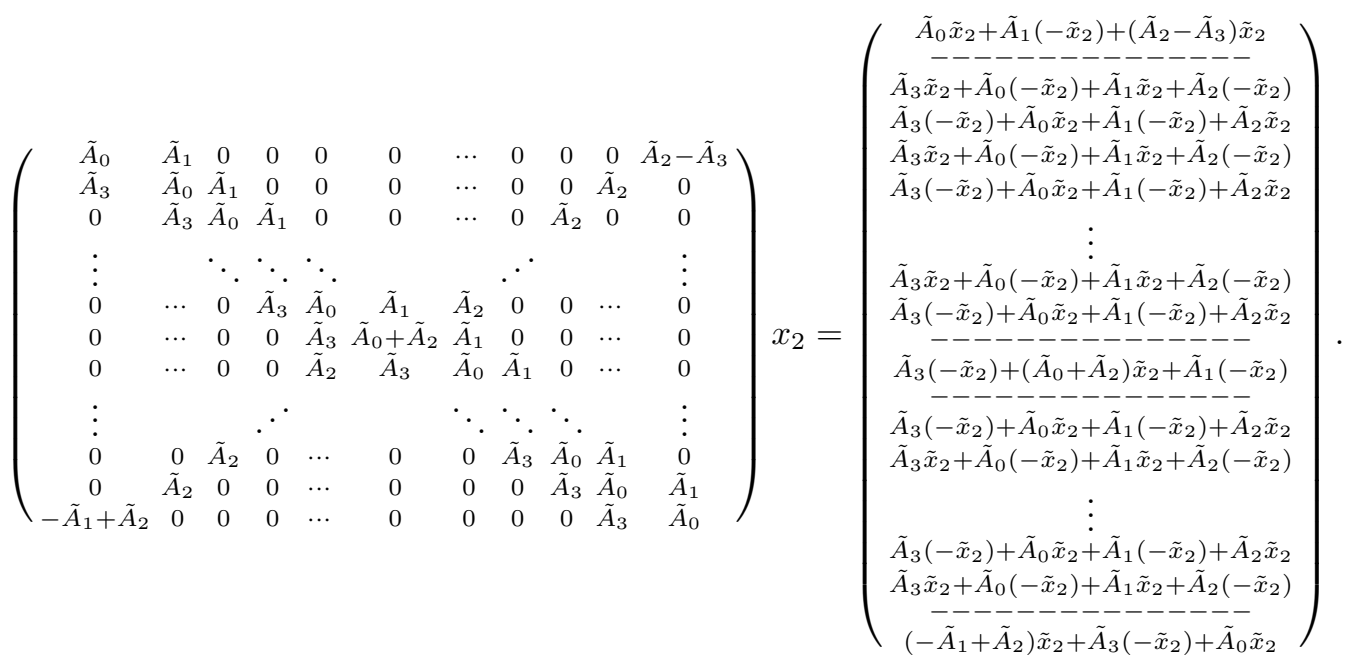

Each entry in the previous vector is $\left(\tilde{A}_{0}-\tilde{A}_{1}+\tilde{A}_{2}-\tilde{A}_{3}\right) \tilde{x}_{2}$ which is zero. This shows that $B_{2} x_{2}=0$. Since the adjacency matrix of $G$ was used to establish $\mathrm{M}(G)=\mathrm{Z}(G)$, by Corollary 1.3 the graph $G$ has field independent minimum rank and $A(G)$ is universally optimal matrix.

\section{REFERENCES}

[1] A. Aazami. Hardness Results and Approximation Algorithms for Some Problems on Graphs. ProQuest LLC, Ann Arbor, MI, 2009. Thesis (Ph.D.)-University of Waterloo (Canada).

[2] A. Aazami. Domination in graphs with bounded propagation: algorithms, formulations and hardness results. J. Comb. Optim., 19(4):429-456, 2010.

[3] AIM Minimum Rank - Special Graphs Work Group (F. Barioli, W. Barrett, S. Butler, S. Cioabă, D. Cvetković, S. Fallat, C. Godsil, W. Haemers, L. Hogben, R. Mikkelson, S. Narayan, O. Pryporova, I. Sciriha, W. So, D. Stevanović, H. van der Holst, K. Vander Meulen, and A. Wangsness). Zero forcing sets and the minimum rank of graphs. Linear Algebra Appl., 428(7):1628-1648, 2008.

[4] J.S. Alameda, E. Curl, A. Grez, L. Hogben, O. Kingston, A. Schulte, D. Young, and M. Young. Families of graphs with maximum nullity equal to zero forcing number. Spec. Matrices, 6:56-67, 2018. 
315 Techniques for determining equality of the maximum nullity and the zero forcing number of a graph

[5] F. Barioli, W. Barrett, S.M. Fallat, H.T. Hall, L. Hogben, B. Shader, P. van den Driessche, and H. van der Holst. Parameters related to tree-width, zero forcing, and maximum nullity of a graph. J. Graph Theory, 72(2):146-177, 2013.

[6] F. Barioli, S. Fallat, and L. Hogben. A variant on the graph parameters of Colin deVerdière: implications to the minimum rank of graphs. Electron. J. Linear Algebra, 13:387-404, 2005.

[7] W. Barrett, A. Francis, and B. Webb. Equitable decompositions of graphs with symmetries. Linear Algebra Appl. 513:409-434, 2017

[8] W. Barrett, H. van der Holst, and R. Loewy. Graphs whose minimal rank is two. Electron. J. Linear Algebra, 11:258-280, 2004.

[9] F. Boesch and R. Tindell. Circulants and their connectivities. J. Graph Theory, 8(4):487-499, 1984.

[10] D. Cvetković, P. Rowlinson, and S. Simić. An Introduction to the Theory of Graph Spectra, vol. 75. London Mathematical Society Student Texts. Cambridge University Press, Cambridge, 2010.

[11] L. Deaett and S.A. Meyer. The minimum rank problem for circulants. Linear Algebra Appl., 491:386-418, 2016.

[12] L.M. DeAlba, J. Grout, L. Hogben, R. Mikkelson, and K. Rasmussen. Universally optimal matrices and field independence of the minimum rank of a graph. Electron. J. Linear Algebra, 18:403-419, 2009.

[13] S. Fallat and L. Hogben. The minimum rank of symmetric matrices described by a graph: a survey. Linear Algebra Appl., 426(2-3):558-582, 2007.

[14] C. Godsil and G. Royle. Algebraic Graph Theory, vol. 207. Graduate Texts in Mathematics. Springer-Verlag, New York, 2001.

[15] A. Gunaratne, L. Hogben, K. In-Jae, B. Kroschel, N. Mcnulty, S. Meyer, S. Narayan, F. Pinero, I. Sciriha, and J. Shen. Aim-isu-cbms circulants, 2013. https://orion.math.iastate.edu/lhogben/10AIM-ISU-CBMS-circulants.pdf

[16] T. Hall. Sage. Code in Sage for determining if a matrix satisfies Strong Arnold Property (SAP), Strong Spectral Property (SSP), Strong Multiplicity Property (SMP), 2016. Sage Worksheet available https://sage.math.iastate.edu/home/ $\mathrm{pub} / 79 /$

[17] L. Hogben, editor. Handbook of Linear Algebra. Discrete Mathematics and its Applications, 2nd edition, CRC Press, Boca Raton, FL, 2014.

[18] L. Hogben, W. Barrett, J. Grout, H. van der Holst, K. Rasmussen, A. Smith, and D. Young. Minimum rank of families, 2019. http://admin.aimath.org/resources/graph-invariants/minimumrankoffamilies/

[19] L. Lovász, M. Saks, and A. Schrijver. Orthogonal representations and connectivity of graphs. Linear Algebra Appl. 114/115:439-454, 1989

[20] L. Lovász, M. Saks, and A. Schrijver. A correction: "Orthogonal representations and connectivity of graphs" [Linear Algebra Appl., 114/115:439-454, 1989; MR0986889 (90k:05095)]. Linear Algebra Appl., 313(1-3):101-105, 2000.

[21] H. van der Holst. Three-connected graphs whose maximum nullity is at most three. Linear Algebra Appl., 429(2-3):625$632,2008$.

[22] D.B. West. Introduction to Graph Theory, 2nd ed. Person Education, New Jersey, 2011.

[23] D. Young. Sage. Code in Sage for generating an Extended Cube Graph, 2019. Sage worksheet available https://sage. math.iastate.edu/home/pub/98/ 